\title{
Stato di conservazione e valore di riferimento favorevole per le popolazioni di uccelli nidificanti in Italia
}

\author{
Marco Gustin $^{1 *}$, Mattia Brambilla ${ }^{2,3}$, Claudio Celada ${ }^{1}$
}

Riassunto - Le linee guida comunitarie per monitorare lo stato di conservazione delle specie e degli habitat richiedono che gli Stati membri forniscano un'indicazione del "Favourable Reference Value" (FRV), o "Valore di Riferimento Favorevole". Il FRV rappresenta un obiettivo di conservazione a lungo termine, tale da rappresentare una situazione indubbiamente favorevole per una data specie, in grado di garantirle ottime possibilità di persistenza nel lungo periodo. La disponibilità di FRV consente una valutazione più oggettiva e trasparente dello stato di conservazione di una specie. Il presente lavoro ha valutato lo stato di conservazione delle specie ornitiche nidificanti in Italia, sviluppando un metodo basato sui requisiti delle direttive comunitarie che integra al suo interno la definizione dei valori di riferimento. Attualmente, è stato proposto un metodo per la definizione dei FRV per popolazione, range e habitat per ciascuna specie, ma è stato possibile procedere ad una identificazione su base quantitativa del solo FRV relativo alla popolazione per le specie di uccelli regolarmente nidificanti in Italia e non attualmente in fase di espansione demografica in seguito a recente colonizzazione (ultimi 30 anni). L'approccio sviluppato per definire il FRV di popolazione ha previsto l'utilizzo di tecniche di Population Viability Analysis o, in alternativa, valutazioni basate sulla densità riproduttiva, secondo le caratteristiche di abbondanza e distribuzione delle specie nidificanti (popolazioni maggiori o minori di 2500 coppie, coloniali o non). Sono state prese in considerazione 250 specie nidificanti in Italia, di cui 88 (che comprendono due sottospecie) incluse nell'Allegato I della Direttiva Uccelli (147/2009CE). Complessivamente, per 46 popolazioni appartenenti a 20 specie inserite nell'Allegato I e per 10 popolazioni di 6 specie non incluse, è stato possibile calcolare un valore di FRV attraverso tecniche di PVA. Per 15 specie inserite nell'Allegato e per 92 specie non inserite è stato formulato un FRV in termini di densità riproduttiva a una o due scale spaziali; per le specie con popolazioni superiori a 2500 coppie esigenze spaziali elevate (territori o home ranges di decine di ettari o più) non è stato formulato il FRV a scala locale. Per valutare lo stato di conservazione è stato utilizzato un adattamento della classificazione a "semaforo" proposta dalla Commissione Europea per la Direttiva Habitat, attribuendo a ciascuna delle tre voci considerate (popolazione, range e habitat), un giudizio sintetico:

${ }^{1}$ Area Conservazione della Natura Lipu, via Udine 3/a, 43100 Parma, Italia.

2 Fondazione Lombardia per l'Ambiente, Settore Biodiversità e Aree protette, Largo 10 luglio 1976 1, 20822 Seveso (MB), Italia.

Museo delle Scienze, Sezione di Zoologia dei Vertebrati, Corso del lavoro e della Scienza 3, 38123 Trento, Italia.

${ }^{3}$ Area Conservazione della Natura Lipu, via Udine 3/a, 43100 Parma, Italia.

* Autore corrispondente: marco.gustin@lipu.it

(C) 2016 Marco Gustin, Mattia Brambilla, Claudio Celada

Received: 22 April 2016

Accepted for publication: 2 August 2016 favorevole: semaforo VERDE. Tutti favorevoli oppure due favorevoli ed uno sconosciuto;

inadeguato: semaforo GIALLO. Uno o più inadeguato/i ma nessuno cattivo;

cattivo: semaforo ROSSO. Uno o più cattivo/i;

sconosciuto semaforo BIANCO. Tre sconosciuti oppure due sconosciuti ed un favorevole.

Prima di poter attribuire il giudizio a ciascuna voce, è necessario verificare se vi sono fattori che possono portare almeno uno dei tre valori di riferimento favorevole a non essere raggiunto, mantenuto o raggiungibile nel futuro prossimo (warning lights). Complessivamente, 42 specie incluse nell'Allegato I della Direttiva Uccelli hanno stato di conservazione cattivo, 39 inadeguato, 6 favorevole e 1 sconosciuto; tra le specie non inserite, 35 hanno stato di conservazione cattivo, 44 inadeguato, 67 favorevole e 16 sconosciuto Per alcune specie è stato possibile valutare lo stato di conservazione per singole bioregioni e sono state prodotte classificazioni "a semaforo" per ciascuna bioregione ospitante la specie in oggetto. Per essere in stato di conservazione favorevole, una specie non deve essere semplicemente al riparo dal rischio di estinzione, ma deve avere un ruolo "significativo" nel proprio habitat di riferimento, rinvenendosi con frequenze e densità soddisfacenti e ricoprendo le funzioni ecologiche che le sono proprie. Le forti pressioni cui molte specie e popolazioni sono sottoposte (cambiamenti climatici, continuo degrado ambientale, variazioni ad ampia scala nella dinamica di popolazione), rendono necessario valutare accuratamente le minacce e pressioni cui la specie/popolazione sono soggette o potranno esserlo nel prossimo futuro, anche in caso di popolazioni superiori al FRV. Risulta, infine, evidente come i FRV dovranno essere sottoposti a periodica rivalutazione e aggiornamento, sulla base soprattutto dei nuovi dati che ogni sei anni vengono forniti dal Reporting sull'applicazione della Direttiva Uccelli.

Parole chiave: Popolazioni Italiane nidificanti, stato di conservazione, FRV (Valore di Riferimento Favorevole), PVA, Population modelling, densità di popolazione.

Abstract - Conservation status and favourable reference value for the bird species breeding in Italy.

EU guidelines for monitoring conservation status of species and habitat require that Member States of the EU provide an indication of the "Favourable Reference Value" (FRV). FRV represents a long-term conservation objective, such as to identify a definitely favourable condition for a species, ensuring long-term persistence. Its use allows for a more thorough and transparent evaluation of the conservation status of a species. This work evaluated the conservation status of bird species breeding in Italy, by developing a method based on EU Directives' requirements and including FRV use. We developed a method for the definition of population, range and habitat FRVs, but until now only population FRVs have been calculated for species regularly breeding in Italy and currently not undergoing population increase after recent colonisation ( $<30$ years). Our approach for population FRV definition involved the use of Population Viability Analysis (PVA) or, alternatively, density-based evaluation, according to distribution and abun- 
dance traits of breeding species (population larger or smaller than 2500 pairs, colonial or non-colonial). We considered 250 breeding species, of which 88 (including two subspecies) listed in the Annex I of the Birds Directive (147/2009CE). Overall, it was possible to calculate a FRV value by means of PVAs for 46 populations belonging to 20 species included in the Annex I of the Bird Directive and for 10 populations of 6 species not included in the Annex. For 15 species listed in the Annex I and for 92 not included, a density-based FRV was provided, at one or two spatial scales; for wide-ranging species with large home ranges or territories, the FRV at the "local" scale was not given. To evaluate the conservation status of the breeding species, the traffic light categories (red: poor, yellow: inadequate, green: favorauble) proposed by Commission for Habitat Directive, were adopted and applied to all the three components considered (population, range and habitat), and an overall synthetic value was provided (favourable, inadequate, poor, unknown) according to the following scheme:

favourable: green. All green or two green and one unkwnow;

inadequate: yellow. One or more yellow but no red;

poor: red. One or more red;

unknown: white. Three unknown or two plus one green.

Before the eventual evaluation of the conservation status for each component, it is essential to check whether there are factors potentially preventing reaching or maintaining FRV in a near future (warning lights). Overall, 42 species listed in the Annex I of the Birds Directive have bad conservation status, 39 inadequate, 6 favourable and 1 unknown. Among species not listed in the Annex I, 35 have bad conservation status, 44 inadequate, 67 favourable and 16 unknown. For some species, the conservation status was also evaluated at the bioregional level, and for each bioregion the traffic light evaluation was given. To be in a favourable conservation status, it is not enough that a species/population is out of extinction risk, as the species should fully play its key ecological functions in its environment. Thus, FRV provided should not be applied automatically and in an uncritical way. The strong pressures acting on many species and populations (climate change, environmental degradation, large-scale changes in population dynamics) require an accurate evaluation of threats and pressures acting on species/populations now or in the next future, also for populations currently at or above the relative FRV. Furthermore, FRVs should be periodically reviewed and updated, with particular reference to the new data provided every six years by the reporting activity carried out within the framework of the Birds Directive.

Key words: Italian breeding population, conservation status, FRV (Favourable Reference Status), PVA, Population modelling, population density.

\section{INTRODUZIONE}

La Direttiva Habitat (92/43/CEE) e la Direttiva Uccelli (2009/147/CE) costituiscono strumenti fondamentali per fermare il declino della biodiversità nei paesi dell'Unione Europea. Le linee guida per l'implementazione della Direttiva Habitat richiedono agli Stati membri di fornire una valutazione dello stato di conservazione per ciascuna specie e habitat, un'indicazione delle tendenze demografiche e un'indicazione del "Favourable Reference Value" (FRV) o "Valore di Riferimento Favorevole". Per quanto riguarda le specie, lo stato di conservazione è considerato soddisfacente se i dati relativi alla popolazione di una specie mostrano una persistenza a lungo termine, la sua abbondanza e distribuzione risultano stabili o in incremento e gli habitat utilizzati dalla specie sono considerati sufficienti per garantire su lungo periodo la persistenza della specie.

Valutare appropriatamente lo stato di conservazione di una specie rappresenta quindi un requisito di basilare importanza non solo per l'adempimento alle richieste comunitarie ma anche, e soprattutto, per pianificare le strategie di conservazione e gestione di specie e habitat.
L'utilizzo dei valori di riferimento favorevole può contribuire ad una valutazione più oggettiva, trasparente e sostanziata dello stato di conservazione di una specie, fornendo dei parametri da utilizzare come termine di confronto.

Secondo i principi della Direttiva Habitat, il FRV rappresenta un obiettivo di conservazione a lungo termine, tale da rappresentare una situazione indubbiamente favorevole per una data specie, in grado di garantirle ottime possibilità di persistenza nel lungo periodo (vedi anche Brambilla et al., 2011). La definizione di un FRV consente di valutare in modo più obiettivo lo stato di conservazione di una specie: più i parametri rilevati sono lontani dai valori di riferimento, più lo stato di conservazione della specie in questione è insoddisfacente. Valori di riferimento possono essere definiti sia per la popolazione, che per l'habitat ed il range di una specie.

All'interno di un percorso pluriennale e con la collaborazione del Ministero dell'Ambiente e della Tutela del Territorio e del Mare e dell'Ispra, abbiamo proposto un approccio per la definizione di valori di riferimento per la popolazione (Brambilla et al., 2011), per l'habitat (Brambilla et al., 2014) e per il range (Brambilla et al., 2012) delle specie ornitiche nidificanti in Italia.

Secondo le linee guida prodotte dal Comitato Habitat, il FRV dovrebbe essere definito da ciascuno Stato membro, per ciascuna specie e per ciascun habitat. Tale definizione deve avvenire su basi tecniche utilizzando i migliori dati disponibili. Sebbene la Direttiva Uccelli non preveda (ancora) esplicitamente l'obbligo della definizione dello stato di conservazione né del valore di riferimento favorevole per ciascuna specie ornitica, da parte degli Stati membri, si ritiene che un'analoga valutazione sia necessaria nell'ambito degli impegni presi per la Strategia EU per la biodiversità fino al 2020, e recentemente la stessa Commissione Europea ha messo a gara uno studio per la definizione di una metodologia per determinare l'FRV nell'ambito della Direttiva Uccelli. Occorre, in ogni caso, analizzare lo stato delle conoscenze (ad es. dati sulla distribuzione e sulla demografia), disponibile per ciascuna specie e valutare la fattibilità della determinazione del FRV per ciascuna delle componenti che concorrono alla definizione dello stato di conservazione.

Con questo lavoro, intendiamo riassumere metodi e risultati del percorso che ha portato alla valutazione dello stato di conservazione delle specie ornitiche nidificanti in Italia, al cui interno si colloca anche la definizione dei metodi per il calcolo dei valori di riferimento per la popolazione. (gli unici per i quali è stato sinora possibile procedere ad un calcolo relativamente esaustivo).

\section{AREA DI STUDIO}

Il presente lavoro ha interessato tutto il territorio italiano, analizzando lo stato della popolazione nazionale delle singole specie nidificanti in Italia. Tuttavia, si è deciso di operare separatamente anche a livello di regioni biogeografiche (alpina, continentale e mediterranea) o di singole popolazioni, quando era possibile individuare chiaramente distinte (meta)popolazioni e tale suddivisione ci è apparsa rilevante per la valutazione dello stato 
di conservazione delle singole specie o per fissare dei target ad un livello spaziale di maggior dettaglio.

\section{MATERIALI E METODI}

L'intero percorso si è articolato secondo le seguenti tappe: ricerca bibliografica; definizione del metodo per il calcolo del FRV; calcolo del FRV di popolazione; valutazione dello stato di conservazione. Successivamente, è stato sviluppato un metodo per la possibile definizione dei valori di FRV per habitat e range.

\section{Ricerca bibliografica}

In primo luogo, è stata effettuata una dettagliata ricerca bibliografica per ottenere informazioni rilevanti per l'areale di distribuzione e la dinamica della popolazione di ciascuna specie, a scala nazionale e per ciascuna regione biogeografica in Italia. La ricerca è partita dall'utilizzo di database elettronici come Web of Science, Biological Abstract, Biosis, Zoological Record, BioOne, per consultare le principali riviste scientifiche di ornitologia, zoologia, ecologia, conservazione. Per quanto concerne i dati italiani, è stato consultato il database elettronico BDO (Banca Dati Ornitologica, http://www.aves.it/bdo2000. $\mathrm{htm})$. Sono state consultate, inoltre, le principali riviste di ornitologia italiane (Rivista Italiana di Ornitologia, Avocetta, Picus, Alula, Uccelli d'Italia) ed internazionali che riguardano popolazioni italiane, gli Atti dei convegni italiani di ornitologia sinora pubblicati. Attraverso la lettura critica dei testi così selezionati, sono stati catalogati in formato elettronico i riferimenti bibliografici utili per la raccolta dei parametri utili all'elaborazione del possibile FRV delle singole specie (successo riproduttivo, mortalità, densità riproduttiva, etc.) e alla valutazione dello stato di conservazione (trend demografico, minacce e pressioni, variazioni areali e di popolazione, qualità dell'habitat, etc.). In totale sono state consultate più di 1900 pubblicazioni. La ricerca bibliografica, soprattutto nel caso di dati su mortalità, successo riproduttivo, etc. è stata ampliata a popolazioni non italiane qualora non esistevano dati soddisfacenti per l'Italia e se le popolazioni considerate potessero essere in qualche misura ecologicamente e demograficamente assimilabili a quelle italiane. La ricerca bibliografica è stata talora integrata da informazioni non pubblicate fornite da esperti ornitologi.

\section{Formulazione del FRV}

Il FRV relativo alla popolazione è stato definito solo per le specie regolarmente nidificanti in Italia (Brichetti \& Fracasso, 2014) e non attualmente in fase di espansione demografica in seguito a recente colonizzazione (ultimi 30 anni), dal momento che per queste ultime risulta difficile prevedere l'esito della colonizzazione o valutare l'entità che tale processo potrebbe raggiungere nel mediolungo periodo.

Per definire il Favourable Reference Value (FRV), l'approccio utilizzato prevede l'utilizzo di tecniche di Population Viability Analysis (PVA) oppure di valutazioni basate sulla densità riproduttiva, a seconda dell'abbondanza e della distribuzione delle specie target (Brambilla et al., 2011). I modelli di PVA possono aiutare a identificare delle popolazioni 'ideali' a seconda delle differenti situazioni immaginate: popolazioni minime vitali (Minimum Viable Population - MVP) per specie o popolazioni ad elevato rischio di estinzione, oppure popolazioni abbastanza ampie da assicurare la persistenza a lungo termine anche in contesti sfavorevoli, nel caso di popolazioni attualmente non a forte rischio di estinzione. Nell'ambito della PVA o population modelling, modelli demografici sono costruiti per migliorare la comprensione delle popolazioni delle specie e/o per affiancare studi di campo nello sviluppo di strategie di conservazione.

Per specie o popolazioni molto abbondanti, le tecniche di PVA incontrano spesso problemi dovuti all'inclusione di grandi numeri di individui, che si traduce in lunghi tempi di analisi, alta variabilità dei risultati con difficoltà di interpretare correttamente gli esiti delle analisi e, soprattutto, in una diminuzione dell'affidabilità delle analisi.

Pertanto, quando i modelli di PVA sono difficilmente utilizzabili, altri parametri di tipo 'demografico' possono aiutare ad identificare target di conservazione o a valutare lo stato di conservazione di una specie o di una popolazione. In particolare, la densità riproduttiva (espressa come numero di individui o coppie o territori o nidi per unità di superficie), può essere un valido indicatore dello stato di conservazione e dell'idoneità ambientale per una data specie in un dato contesto areale/geografico (Gustin et al., 2009; 2010a; 2010b).

È stata operata una suddivisione tra specie riguardo: i) abbondanza a livello nazionale; ii) caratterizzazione biogeografica (bioregione alpina, continentale e mediterranea).

Per quanto riguarda l'abbondanza le specie sono state suddivise in due categorie.

a) le specie con meno di 2.500 coppie nidificanti sono state suddivise in:

a1) specie con singola popolazione: un unico valore di FRV per la popolazione nazionale;

a2) specie con popolazioni frammentate: areale strutturato in unità distinte e con verosimile scarso flusso genico tra esse (popolazioni o colonie riproduttive o gruppi di colonie): valore di FRV specifico per ciascuna unità di areale/popolazione della specie (la conservazione di tali specie è probabilmente dipendente dalla conservazione delle principali popolazioni isolate).

b) le specie con più di 2.500 coppie nidificanti sono state suddivise in:

b1) specie con areale diviso in unità discrete (popolazioni o colonie riproduttive o gruppi di colonie) con popolazioni inferiori a 2.500 coppie: FRV specifico per ciascuna unità di areale/popolazione della specie;

b2) specie con range ampio e più o meno continuo (tutte o la maggior parte delle possibili unità di areale/ popolazioni o colonie o gruppi di colonie della specie più grandi di 2.500 coppie):

b2a) specie non coloniali: FRV espresso in termini di densità riproduttiva a differenti scale spaziali;

b2b) specie coloniali: FRV non formulato.

Il valore soglia di 2500 coppie come limite per il cal- 
colo del FRV a scala nazionale o di regione biogeografica è stato dettato dalla ricerca di un compromesso tra possibilità di analisi affidabili tramite population modelling, garantite per popolazioni ridotte ma più difficili per popolazioni ampie, e necessità di considerare quante più specie possibili, in modo da poter dare indicazioni quantitative, ragionevolmente affidabili, sul FRV.

Per le specie coloniali non è stato formulato un FRV espresso come densità riproduttiva, dal momento che esse tendono a concentrarsi in poco spazio e che la loro abbondanza per unità di superficie riflette solo in piccola parte l'idoneità ambientale più generale, essendo maggiormente influenzata da altri fattori ed in particolare dalla disponibilità di siti idonei alla nidificazione nella tipologia ambientale su cui è insediata la colonia e dalla disponibilità trofica in aree circostanti (su superfici variabili da specie a specie e da sito a sito per la stessa specie).

Per il calcolo del FRV come dimensione di popolazione (abbondanza), come richiesto nei casi a1, a2, b1, è necessario disporre di una serie di parametri demografici e di successo riproduttivo, di fondamentale importanza per analisi secondo tecniche di population modelling. Il FRV per queste specie è stato quindi calcolato qualora fossero noti o stimabili i principali parametri necessari, oppure quando vi fossero stime di tali parametri disponibili per specie "affini", o strettamente imparentate, con ecologia simile e densità comparabile negli habitat d'elezione. Nel caso di specie per le quali non fossero noti i principali parametri richiesti, né vi fossero dati relativi a specie strettamente imparentate, non è stato possibile calcolare il FRV. Quando alcuni dei parametri richiesti dal modelling non erano conosciuti per le specie considerate, sono state condotte simulazioni variando i parametri sconosciuti all'interno dei valori noti per specie affini.

Le analisi di population modelling sono state condotte utilizzando il programma Vortex versione 9.72 (Lacy et al., 2009), comunemente utilizzato per valutare il rischio di estinzione di una popolazione.

Per le specie comprese nella categoria b2 (>2.500 coppie), senza evidente frammentazione/ suddivisione delle popolazioni in unità discrete e non coloniali (b2a), stante la difficoltà (se non l'impossibilità) di ottenere stime affidabili per molte delle specie con popolazioni relativamente numerose, nonché l'inaffidabilità delle analisi condotte tramite population modelling per popolazioni molto ampie, sono stati considerati, ove disponibili, valori di densità riproduttiva a due scale spaziali: locale $(<100$ ha) e di comprensorio (1.000-10.000 ha). Per le specie con esigenze spaziali elevate (territori o home ranges di decine di ettari o più) non è stato formulato il FRV a scala locale (es. Calandra Melanocorypha calandra). In alcuni casi, soprattutto per specie ecotonali che verosimilmente occorrono in tessere di ambiente idoneo sparse in una matrice meno idonea, il FRV è stato ulteriormente suddiviso in casi differenti legati a diversa idoneità ambientale (es. Occhiocotto Sylvia melanocephala).

L'utilizzo di valori di densità riproduttiva ricavati da popolazioni ritenute in stato di conservazione ottimo o soddisfacente in ambienti idonei alle esigenze ecologiche della specie in oggetto, consente di verificare, tramite il confronto dei valori di densità rilevati all'interno di un determinato sito con i valori di riferimento, lo stato di conservazione della specie in quel determinato contesto.

Le scale considerate dovrebbero rappresentare i livelli più opportuni per valutare lo stato di conservazione di una specie attraverso la sua densità nelle ZPS, poiché l'estensione di queste ultime varia da pochi ha a diverse migliaia di ha: solo le ZPS di maggiori dimensioni superano i 100.000 ha di estensione, ma includono al loro interno habitat eterogenei con differenti comunità ornitiche e differenti gradi di idoneità per le specie considerate.

L'utilizzo di valori di densità diventa così uno strumento operativo per valutare lo stato di salute di una popolazione e trarne le debite considerazioni nell'ambito dei programmi di gestione e conservazione di habitat e specie.

La definizione del FRV basata su valori di densità riproduttiva ha un valore 'parziale', poiché esso può essere utilizzato agevolmente come termine di riferimento per singoli siti o gruppi di siti (i valori di FRV basati su densità sono formulati in modo da simulare le principali condizioni riscontrabili nelle ZPS), ma non danno un termine di confronto direttamente applicabile all'intera scala nazionale. Sforzi futuri dovrebbero cercare di tradurre questo valore di densità in stime di popolazione, sulla base del range attuale, storico e/o potenziale di ciascuna specie; tuttavia, tale lavoro richiede una quantificazione dell'habitat potenzialmente idoneo alle diverse specie che attualmente non è disponibile (Brambilla et al., 2013).

Nella formulazione presente, in alcuni casi i valori di densità su cui si basa il FRV, prevedono valori differenti per aree più o meno idonee alle esigenze ecologiche della specie. La necessità di questa distinzione (ulteriore rispetto a quella tra le due scali spaziali), è legata alla presenza di specie che occupano spesso 'tessere' di ambienti relativamente isolati all'interno di matrici di habitat non idonee o poco idonee: in questo caso, si è ritenuto utile fornire un valore di densità (più basso) per situazioni con presenza sparsa di ambienti idonei alla specie considerata e un valore di densità (più alto) relativo invece a estensioni continue di habitat adatto alle esigenze ecologiche della specie in questione.

Per ulteriori dettagli relativi alla procedura adottata per la definizione del FRV si rimanda a Brambilla et al. (2011).

Valutazione dello stato di conservazione e classificazione 'a semaforo'

La metodologia proposta tiene conto delle indicazioni fornite dall"'Habitat Committee" nel documento DocHab04-03 "Assessment, monitoring and reporting under Art 17 of the Habitat Directive", mirando ad adattare tali linee guida all'avifauna italiana (Evans \& Arvela, 2011).

È del tutto prevedibile che per la maggior parte delle specie, le informazioni richieste nel documento di cui sopra saranno solo parzialmente esistenti o, in alcuni casi, non disponibili. L'approccio di questa proposta è di aderire comunque il più possibile al formato proposto per la Direttiva Habitat, calando tale impostazione nella realtà dei dati disponibili. 
La classificazione a "semaforo" (rosso, giallo, verde, bianco) proposta dalla Commissione per la Direttiva Habitat e da noi specificatamente riadattata per l'avifauna italiana, è stata già adottata in uno studio comparativo dei fattori potenzialmente influenzanti lo stato di conservazione delle specie ornitiche italiane (Brambilla et al., 2013).

$\mathrm{Nel} 2005$, il Comitato Habitat ha definito le linee guida per monitorare lo stato di conservazione delle specie e degli habitat tutelati dalla Direttiva Habitat (43/92/EU). Lo stato di conservazione di una specie può essere considerato soddisfacente se:

la popolazione mostra persistenza a lungo termine;

la sua abbondanza e distribuzione risultano stabili o in incremento;

gli habitat utilizzati sono considerati sufficienti per garantire la persistenza della specie nel lungo periodo.

Gli elementi da considerare sono relativi a:

popolazione;

range;

habitat;

prospettive future (l'elemento di "prognosi" forma parte integrale dei risultati della valutazione).

Nel caso della popolazione, oggetto di questo lavoro, $\mathrm{i}$ criteri per l'attribuzione del giudizio sono risultati:

favorevole: popolazione ritenuta stabile (o in espansione), popolazioni non inferiori al relativo FRV (quando noto come dimensione di popolazione) e parametri riproduttivi, mortalità e struttura di età che non deviano dai valori normali (se disponibili); se non sono disponibili i dati di popolazione e FRV non è possibile dichiarare la popolazione in stato favorevole (per popolazioni nazionali o bioregionali fino a 2.500 coppie);

inadeguato: popolazione in declino per meno del 10\% in 10 anni o inferiore al FRV (quando noto come dimensione di popolazione) (ma superiore al $75 \%$ del FRV); popolazione non in calo ma ridotta (inferiore a un verosimile valore di FRV) oppure sensibile a vistose fluttuazioni a breve periodo senza trend generale evidente;

cattivo: popolazione in declino di oltre il $10 \%$ in 10 anni e inferiore al FRV (quando noto come dimensione di popolazione) o inferiore di almeno il $25 \%$ rispetto al FRV (quando noto come dimensione di popolazione) o riproduzione, mortalità e struttura di età che deviano fortemente dai valori normali (se dati disponibili); popolazione non in calo ma estremamente ridotta.

È stata attribuita la qualifica 'sconosciuto' a quelle voci per le quali non si disponeva, al momento della trattazione, di informazioni sufficienti (ed attendibili) per valutare la popolazione della singola specie.

Prima di poter attribuire il giudizio (ed in particolare la valutazione "favorevole") a ciascuna delle voci valutate, è necessario verificare se vi sono fattori che con buona probabilità possono portare almeno uno dei tre valori di riferimento favorevole a non essere raggiunto, mantenuto o raggiungibile nel futuro prossimo ('warning lights'). Questi fattori non sono utilizzati direttamente nella definizione dei FRV, ma sono fattori che hanno già un impatto o che potranno averlo verosimilmente in futuro. Tali elementi devono essere definiti sulla base del problema che può impedire il raggiungimento del FRV.
Per quanto riguarda la popolazione, abbiamo individuato i seguenti fattori potenzialmente critici: struttura di popolazione (classi di età, sex ratio) che devia fortemente dalla struttura attesa per popolazioni stabili/in salute (stato di conservazione "inadeguato" o "cattivo"), successo riproduttivo o tasso di sopravvivenza o altro parametro demografico che devia fortemente dalla struttura attesa per popolazioni stabili/in salute (stato di conservazione "inadeguato" o "cattivo"), popolazione non inferiore a FRV ma in calo: popolazione in calo: $<10 \%$ in 10 anni ("inadeguato"), in calo $>10 \%$ in 10 anni ("cattivo").

Per quanto riguarda l'habitat, si deve valutare se una riduzione dell'habitat idoneo alla specie (valutata rispetto al FRV-H) possa essere molto probabile in futuro a causa di trasformazioni del territorio, distruzione di habitat d'importanza cruciale, cambiamenti climatici, etc.

Nel caso del range, il principale elemento di criticità in questo senso riguarda una verosimile diminuzione del range al di sotto del FRV-R a causa di fattori quali disturbo persistente o caccia che possono ridurre l'areale della specie anche in assenza di variazioni significative nella disponibilità di habitat idoneo, cambiamenti climatici che possono compromettere l'idoneità di intere aree per una specie, etc. Inoltre, anche un range particolarmente ridotto e/o frammentato rappresenta chiaramente un fattore di potenziale criticità.

È stata utilizzata la classificazione a "semaforo" (rosso, giallo, verde, bianco) proposta dalla Commissione per la Direttiva Habitat, attribuendo a ciascuna delle tre voci considerate un giudizio sintetico: favorevole, cattivo, inadeguato, sconosciuto. Si è poi calcolato il valore complessivo dello stato di conservazione secondo il criterio sotto riportato e derivato dalla proposta della commissione:

favorevole: semaforo VERDE: tutti favorevoli oppure due favorevoli ed uno sconosciuto;

inadeguato: semaforo GIALLO: uno o più inadeguato/i ma nessuno cattivo;

cattivo: semaforo ROSSO: uno o più cattivo/i;

sconosciuto: tre sconosciuti oppure due sconosciuti ed un favorevole.

Per alcune specie è stato possibile valutare lo stato di conservazione per singole bioregioni e sono state prodotte classificazioni 'a semaforo' per ciascuna bioregione ospitante la specie in oggetto.

\section{RISULTATI}

La formulazione del FRV (Gustin et al., 2009; 2010a; 2010b; Brambilla et al., 2011) è stata effettuata qualora la informazioni disponibili siano state ritenute sufficienti sia per quanto riguarda la presenza storica in Italia (specie nidificanti da almeno trent'anni), che per la relativa distribuzione e abbondanza delle specie.

Le specie sono state affrontate in ordine sistematico, secondo quanto riportato da Fracasso et al. (2009).

Sono state prese in considerazione 250 specie nidificanti in Italia, di cui 88 (che comprendono due sottospecie) incluse nell'allegato I della Direttiva Uccelli e 162 non incluse. 
Il livello biogeografico (scala alpina, continentale e mediterranea), è stato analizzato per 8 specie (16 popolazioni) dell'Allegato I della Direttiva Uccelli e 34 specie (73 popolazioni) non inserite nell'Allegato I.

Complessivamente, sono stati formulati valori di FRV per 35 specie incluse nell'Allegato I (Tab. 1) e per 101 specie non inserite nell'Allegato (Tab. 2).

In Appendice sono riportati i risultati riguardanti la formulazione del FRV per singola specie e i dettagli rela- tivamente all'analisi della PVA o della densità delle singole specie a scala locale o di comprensorio.

La valutazione dello stato di conservazione delle singole specie è stata fatta considerando separatamente per range, popolazione, habitat, che convergono a determinare il valore complessivo secondo quanto descritto nei Metodi (Tab. 3 per le specie in Allegato I della Direttiva Uccelli, Tab. 4 per quelle non inserite).

Tab. 1 - Riepilogo dei valori di FRV per le specie inserite nell'Allegato I della Direttiva Uccelli. / Summary of FRV values for the species included in the Annex I of the Birds Directive.

\begin{tabular}{|c|c|}
\hline Specie & FRV \\
\hline Uccello delle tempeste & $\begin{array}{l}\text { Sicilia: } 3.000 \text { individui ( } 1.000 \text { coppie }) \\
\text { Sardegna: } 3.000 \text { individui ( } 1.000 \text { coppie })\end{array}$ \\
\hline Marangone dal ciuffo & $\begin{array}{l}\text { Tirreno centrale: } 290 \text { individui ( } 75-80 \text { coppie }) \\
\text { Sicilia: } 290 \text { individui ( } 75-80 \text { coppie }) \\
\text { Sardegna: } 9.000 \text { individui }(2.120 \text { coppie })\end{array}$ \\
\hline Cicogna bianca & $\begin{array}{l}\text { Italia nord-occidentale: } \\
220-230 \text { coppie ( } 900 \text { individui) con successo riproduttivo non inferiore al } 50 \%\end{array}$ \\
\hline Nibbio bruno & $\begin{array}{l}\text { Area prealpino-padana: } 700 \text { coppie }(1.700 \text { individui) } \\
\text { Italia peninsulare: } 700 \text { coppie ( } 1.700 \text { individui) }\end{array}$ \\
\hline Nibbio reale & $\begin{array}{l}\text { Italia peninsulare: } 670 \text { coppie ( } 1.700 \text { individui) } \\
\text { Sicilia: } 30 \text { coppie ( } 80 \text { individui) con } 60 \% \text { degli adulti che si riproduce con successo ogni } \\
\text { anno e tasso d'involo } \geq 1.5 \text { (oppure } 50 \% \text {, con tasso d'involo } \geq 1.7 \text { ) } \\
\text { Sardegna: } 30 \text { coppie }(80 \text { individui) con } 60 \% \text { degli adulti che si riproduce con successo } \\
\text { ogni anno e tasso d'involo } \geq 1.5 \text { (oppure } 50 \% \text {, con tasso d'involo } \geq 1.7 \text { ) }\end{array}$ \\
\hline Capovaccaio & $\begin{array}{l}40 \text { coppie ( } 132 \text { individui) con } 78 \% \text { degli adulti che si riproduce con successo, tasso d'involo } \\
1.4 ; \text { o } 30 \text { coppie ( } 102 \text { individui), con } 90 \% \text { degli adulti che si riproduce con successo, tasso } \\
\text { d'involo } 1.4\end{array}$ \\
\hline Falco di palude & 700 coppie (2500 individui) \\
\hline Albanella minore & $\begin{array}{l}\text { Pop. padano-adriatica: } 300 \text { coppie (1.000 individui) } \\
\text { Popolazione tirrenica: } 300 \text { coppie (1.000 individui) }\end{array}$ \\
\hline Aquila reale & $\begin{array}{l}\text { Alpi: } 410 \text { coppie ( } 1.100 \text { individui) } \\
\text { Appennini: } 170 \text { coppie ( } 400 \text { individui) } \\
\text { Sicilia: } 20 \text { coppie } \\
\text { Sardegna: } 55 \text { coppie }\end{array}$ \\
\hline Aquila di Bonelli & 200-210 coppie (600 individui); vedi testo \\
\hline Falco della regina & $\begin{array}{l}\text { Sardegna: } 900 \text { coppie }(2200 \text { individui) } \\
\text { Sicilia: } 320 \text { coppie }(800 \text { individui) con produttività } \geq 1.26 \text { giovani per coppia }\end{array}$ \\
\hline Lanario & $\begin{array}{l}\text { Italia peninsulare: } 265 \text { coppie ( } 630 \text { individui) } \\
\text { Sicilia: } 170 \text { coppie ( } 420 \text { individui) }\end{array}$ \\
\hline Falco pellegrino & $\begin{array}{l}\text { Alpi e Prealpi: } 500 \text { coppie } \\
\text { Italia peninsulare: } 350 \text { coppie } \\
\text { Sicilia: } 250 \text { coppie } \\
\text { Sardegna: } 200 \text { coppie }\end{array}$ \\
\hline Francolino di monte & $\begin{array}{l}\text { a scala di comprensorio: } 3 \text { coppie ( } 4 \text { per aree particolarmente vocate) per } 100 \text { ha } \\
\text { a scala locale: } 6-10 \text { coppie per } 100 \text { ha (a seconda dell'idoneità dell'habitat) }\end{array}$ \\
\hline Pernice bianca & $\begin{array}{l}10 \text { maschi per } 10 \mathrm{~km}^{2} \text { a scala di comprensorio } \\
4-5 \text { maschi per } 100 \text { ha a scala locale }\end{array}$ \\
\hline Fagiano di monte & $\begin{array}{l}\text { a scala di comprensorio: } 10 \text { individui per } \mathrm{km}^{2} \\
\text { a scala locale: } 20 \text { individui per } 100 \text { ha (densità a fine estate) }\end{array}$ \\
\hline
\end{tabular}




\begin{tabular}{|c|c|}
\hline Specie & FRV \\
\hline Gallo cedrone & $\begin{array}{l}\text { a scala di comprensorio: } 4 \text { individui per } \mathrm{km}^{2} \\
\text { a scala locale: } 6 \text { individui per } 100 \text { ha (densità a fine estate) }\end{array}$ \\
\hline Pernice sarda & a scala locale: 6-7 coppie per 100 ha oppure densità autunnale di 40 individui per 100 ha \\
\hline Avocetta & $\begin{array}{l}\text { popolazione adriatica: } 1.850-1.860 \text { coppie ( } 4.400 \text { individui) } \\
\text { Sardegna: } 600 \text { coppie ( } 1.470 \text { individui) } \\
\text { Sicilia: } 400 \text { coppie ( } 980 \text { individui) }\end{array}$ \\
\hline Fratino & $\begin{array}{l}\text { popolazione nord-adriatica: } 500 \text { coppie } \\
\text { popolazione meridionale: } 500 \text { coppie } \\
\text { popolazione tirrenica: } 500 \text { coppie } \\
\text { Sicilia: } 500 \text { coppie } \\
\text { Sardegna: } 500 \text { coppie }\end{array}$ \\
\hline Sterna zampenere & $\begin{array}{l}\text { popolazione continentale: } 570 \text { coppie ( } 2.000 \text { individui) } \\
\text { Sardegna: } 200 \text { coppie ( } 720 \text { individui) }\end{array}$ \\
\hline Sterna comune & Sardegna: 1.050 coppie (3.150-3.200 individui) \\
\hline Civetta capogrosso & a scala di comprensorio: 1.5 coppia per $\mathrm{km}^{2}$ \\
\hline Succiacapre & $\begin{array}{l}\text { ambienti solo parzialmente idonei: a scala di comprensorio: } 1 \text { coppia per } \mathrm{km}^{2} \text {; a scala } \\
\text { locale: } 5 \text { coppie per } 100 \text { ha } \\
\text { ambienti ampiamente vocati: a scala di comprensorio: } 5 \text { coppie per } \mathrm{km}^{2} \text {; a scala locale: } 20 \\
\text { coppie per } 100 \text { ha }\end{array}$ \\
\hline Martin pescatore & coppia per km di corso d'acqua; una coppia per 10 ha in zone umide \\
\hline Calandra & $\begin{array}{l}\text { a scala di comprensorio: } 10 \text { coppie per } \mathrm{km}^{2} \\
\text { a scala locale: } 6 \text { coppie per } 10 \text { ha }\end{array}$ \\
\hline Calandrella & $\begin{array}{l}\text { a scala di comprensorio: } 20 \text { coppie per } \mathrm{km}^{2} \text { ( } 2 \text { coppie per } \mathrm{km}^{2} \text { in aree solo parzialmente } \\
\text { idonee) } \\
\text { a scala locale: } 10 \text { coppie per } 10 \text { ha }\end{array}$ \\
\hline Tottavilla & $\begin{array}{l}\text { a scala di comprensorio: } 10 \text { coppie per } \mathrm{km}^{2} \\
\text { a scala locale: } 3 \text { coppie per } 10 \text { ha }\end{array}$ \\
\hline Calandro & $\begin{array}{l}\text { a scala di comprensorio: } 10 \text { coppie per } \mathrm{km}^{2} \\
\text { a scala locale: } 2-3 \text { coppie per } 10 \mathrm{ha}\end{array}$ \\
\hline Magnanina comune & $\begin{array}{l}\text { a scala di comprensorio: } 50 \text { coppie per } \mathrm{km}^{2} \text { (provvisorio) } \\
\text { a scala locale: } 12-13 \text { coppie per } 10 \text { ha (brughiere dell'entroterra); } 20 \text { coppie per } 10 \text { ha } \\
\text { (macchia costiera fitta e relativamente bassa) }\end{array}$ \\
\hline Bigia padovana & 4.000 coppie (8.000 individui) \\
\hline Balia dal collare & $\begin{array}{l}\text { Alpi e Prealpi italo-elvetiche: } 3.000 \text { coppie ( } 6.000 \text { individui) } \\
\text { Appennini: } 3.000 \text { coppie ( } 6.000 \text { individui) }\end{array}$ \\
\hline Averla piccola & $\begin{array}{l}\text { a scala di comprensorio: } 1 \text { coppia per } \mathrm{km}^{2} \text { (ambienti a mosaico), } 5 \text { coppie per } \mathrm{km}^{2} \text { (vaste } \\
\text { aree aperte o semi-aperte) } \\
\text { a scala locale: } 0.5 \text { coppie per } 10 \text { ha (ambienti a mosaico), } 5 \text { coppie per } 10 \text { ha (ambienti aperti) } \\
\text { in aree particolarmente idonee: } 10 \text { coppie per } 10 \text { ha }\end{array}$ \\
\hline Gracchio corallino & $\begin{array}{l}\text { Alpi: } 8.000 \text { coppie }(25.000 \text { individui }) \\
\text { Appennini: } 8.000 \text { coppie }(25.000 \text { individui }) \\
\text { Sicilia: } 1.000 \text { coppie }(2.500 \text { individui }) \\
\text { Sardegna } 1.000 \text { coppie }(2.500 \text { individui })\end{array}$ \\
\hline Ortolano & $\begin{array}{l}\text { a scala di comprensorio: } 10 \text { coppie per } \mathrm{km}^{2} \text { (vedi testo) } \\
\text { a scala locale: } 8 \text { coppie per } 10 \text { ha (una coppia per ha in aree particolarmente idonee di } \\
\text { pochi ettari) }\end{array}$ \\
\hline
\end{tabular}


Tab. 2 - Riepilogo dei valori di FRV per le specie non inserite nell'Allegato I della Direttiva Uccelli. / Summary of FRV values for the species not included in the Annex I of the Birds Directive.

\begin{tabular}{|c|c|}
\hline Specie & FRV \\
\hline Germano reale & a scala di comprensorio: 5 nidi per $\mathrm{km}^{2}$ \\
\hline Starna & $\begin{array}{l}\text { popolazione padana - nord-appenninica: } 10 \text { coppie per } \mathrm{km}^{2}(20 \text { per il Mezzano }) \text { in primavera } \\
\text { e } 25 \text { individui per } \mathrm{km}^{2} \text { in tarda estate }\end{array}$ \\
\hline Quaglia & 10 maschi in canto per 100 ha a scala locale (vedi testo) \\
\hline Cormorano & Sardegna: 190 individui (60 coppie) \\
\hline Tuffetto & a scala locale: 6 coppie per 10 ha \\
\hline Astore & $\begin{array}{l}\text { area alpina-padana: } 1900 \text { individui ( } 800 \text { coppie) } \\
\text { area appenninico-mediterranea: } 1900 \text { individui ( } 800 \text { coppie) }\end{array}$ \\
\hline Sparviere & 20 coppie per $100 \mathrm{~km}^{2} ; 5$ coppie per $100 \mathrm{~km}^{2}$ per contesti sub-ottimali \\
\hline Poiana & $\begin{array}{l}\text { a scala di comprensorio: } 30 \text { coppie per } 100 \mathrm{~km}^{2} \text { per contesti molto idonei, } 20 \text { coppie per } \\
100 \mathrm{~km}^{2} \text { per contesti sub-ottimali }\end{array}$ \\
\hline Gheppio & $\begin{array}{l}\text { a scala di comprensorio: } 50 \text { coppie per } 100 \mathrm{~km}^{2} \text { in ambienti favorevoli (paesaggi aperti e } \\
\text { semi-aperti), } 25 \text { coppie per } 100 \mathrm{~km}^{2} \text { in aree meno vocate } \\
\text { a scala locale: } 5-10 \text { coppie per } \mathrm{km}^{2}\end{array}$ \\
\hline Lodolaio & $\begin{array}{l}\text { popolazione padana-alpina-alto adriatica: } 1000 \text { coppie } \\
\text { regioni tirreniche e centro-meridionali: } 1800 \text { individui ( } 700 \text { coppie) } \\
\text { Sardegna: } 190 \text { individui ( } 75 \text { coppie) } \\
\text { Sicilia: } 190 \text { individui ( } 75 \text { coppie) }\end{array}$ \\
\hline Porciglione & $\begin{array}{l}\text { a scala locale: } 10 \text { coppie per } 10 \text { ha per zone umide con presenza di canneti e/o tifeti } \\
3 \text { coppie per ha per canneti e canneti/tifeti di piccole dimensioni, continui ed omogenei }\end{array}$ \\
\hline Gallinella d'acqua & $\begin{array}{l}7 \text { coppie per km lungo corsi d'acqua (fiumi, canali) } \\
5 \text { coppie per ha per zone umide piccole o medio-piccole (in aree umide di dimensioni molto } \\
\text { piccole la densità può essere anche più elevata) } \\
\text { per zone umide di decine di ettari: } 2 \text { coppie per ha }\end{array}$ \\
\hline Folaga & $\begin{array}{l}6 \text { coppie per ha per zone umide di qualche decina di ettari } \\
8 \text { nidi per km lungo fiumi o grandi canali }\end{array}$ \\
\hline Corriere piccolo & $\begin{array}{l}\text { a scala locale: } 4 \text { coppie per km lungo corsi d'acqua; } 1 \text { coppia per ha in habitat molto } \\
\text { idonei }\end{array}$ \\
\hline Pavoncella & $\begin{array}{l}\text { scala locale: } 0,2 \text { coppie per } 10 \text { ha } \\
\text { scala di comprensorio: } 6 \text { coppie per } \mathrm{km}^{2} \\
\text { scala intermedia (poche centinaia di ettari): } 30 \text { coppie per } \mathrm{km}^{2}\end{array}$ \\
\hline Pettegola & $\begin{array}{l}\text { popolazione alto-adriatica: } 2000 \text { coppie } \\
\text { Sardegna: } 2700 \text { individui (1350 coppie) (vedi testo) }\end{array}$ \\
\hline Piro-piro piccolo & 4000 individui (1600 coppie) \\
\hline Piccione selvatico & 500 coppie per ciascuna delle popolazioni continentali della specie \\
\hline Colombaccio & $\begin{array}{l}\text { a scala locale: } 2 \text { coppie per } 10 \text { ha } \\
\text { a scala di comprensorio: } 4 \text { coppie per } \mathrm{km}^{2}\end{array}$ \\
\hline Tortora dal collare & $\begin{array}{l}\text { a scala locale: } 20 \text { coppie per } 10 \text { ha } \\
\text { a scala di comprensorio: } 50 \text { coppie per } \mathrm{km}^{2}\end{array}$ \\
\hline Tortora selvatica & $\begin{array}{l}\text { a scala locale: } 0.5 \text { coppie per } 10 \text { ha } \\
\text { a scala di comprensorio: } 1 \text { coppia per } \mathrm{km}^{2}\end{array}$ \\
\hline Barbagianni & $\begin{array}{l}\text { bioregioni continentale e alpina: a scala di comprensorio: } 10 \text { coppie per } 100 \mathrm{~km}^{2} \\
\text { bioregione mediterranea: a scala locale: } 1 \text { coppia per } \mathrm{km}^{2} \\
\text { a scala di comprensorio: } 20 \text { coppie per } 100 \mathrm{~km}^{2}\end{array}$ \\
\hline
\end{tabular}




\begin{tabular}{|c|c|}
\hline Specie & FRV \\
\hline Assiolo & $\begin{array}{l}\text { a scala locale: } 6 \text { coppie per } \mathrm{km}^{2} \\
\text { a scala di comprensorio: } 50 \text { coppie per } 100 \mathrm{~km}^{2} \text { per le bioregioni continentale e alpina e } 70 \\
\text { coppie per } 100 \mathrm{~km}^{2} \text { per quella mediterranea }\end{array}$ \\
\hline Civetta & $\begin{array}{l}\text { a scala di comprensorio: } 15 \text { coppie per } 10 \mathrm{~km}^{2} \\
\text { a scala locale: } 1 \text { coppia per } 10 \text { ha per contesti particolarmente idonei }\end{array}$ \\
\hline Allocco & $\begin{array}{l}\text { a scala di comprensorio: } 3 \text { coppie per } \mathrm{km}^{2} \text { per ambienti forestali, } 1.3 \text { coppie per } \mathrm{km}^{2} \text { per } \\
\text { ambienti prevalentemente agricoli }\end{array}$ \\
\hline Gufo comune & a scala di comprensorio: 1.5 coppie per $\mathrm{km}^{2}$ \\
\hline Upupa & $\begin{array}{l}\text { scala di comprensorio: } 50 \text { coppie per } 100 \mathrm{~km}^{2} \\
\text { scala locale: } 2 \text { coppie per } 10 \text { ha }\end{array}$ \\
\hline Torcicollo & $\begin{array}{l}\text { a scala di comprensorio: } 5 \text { coppie per } \mathrm{km}^{2}, 1 \text { coppia per } \mathrm{km}^{2} \text { negli ambienti intensivamente } \\
\text { coltivati }\end{array}$ \\
\hline Picchio verde & a scala di comprensorio: 3 coppie per $\mathrm{km}^{2}$ \\
\hline Picchio rosso maggiore & $\begin{array}{l}\text { a scala di comprensorio: } 5 \text { coppie per } \mathrm{km}^{2}, 0.5 \text { coppia per } \mathrm{km}^{2} \text { negli ambienti intensivamente } \\
\text { coltivati; } \\
\text { a scala locale: } 2 \text { coppie per } 10 \mathrm{ha}\end{array}$ \\
\hline Picchio rosso minore & $\begin{array}{l}\text { a scala di comprensorio: } 1.5 \text { coppie per } \mathrm{km}^{2} \text { per aree mediamente idonee, } 5 \text { coppie per } \mathrm{km}^{2} \\
\text { per aree vaste particolarmente vocate } \\
\text { a scala locale: } 2 \text { coppie per } 10 \mathrm{ha}\end{array}$ \\
\hline Cappellaccia & $\begin{array}{l}\text { scala locale: } 5 \text { coppie per } 10 \text { ha a scala locale ( } 7-8 \text { coppie per } 10 \text { ha per aree particolarmente } \\
\text { idonee) }\end{array}$ \\
\hline Allodola & $\begin{array}{l}\text { per vasti ambienti aperti (copertura della vegetazione erbacea }>75 \% \text { della superficie } \\
\text { complessiva dell'area): } \\
\text { a scala di comprensorio: } 50 \text { coppie per } \mathrm{km}^{2} \\
\text { a scala locale: } 3 \text { coppie per ha a scala più ampia possano essere ritenute soddisfacenti; tali } \\
\text { valori vengono pertanto proposti come FRV } \\
\text { per ambienti aperti più ridotti o semi-aperti (vegetazione erbacea con copertura compresa } \\
\text { tra } 50 \% \text { e } 75 \% \text { dell'area): } \\
\text { a scala locale: } 1 \text { coppia per ha } \\
\text { a scala di comprensorio: } 10 \text { coppie per } \mathrm{km}^{2}\end{array}$ \\
\hline Prispolone & $\begin{array}{l}\text { scala locale: } 4 \text { coppie per } 10 \text { ha (in ambienti particolarmente favorevoli alla specie } 6 \text { coppie } \\
\text { per } 10 \mathrm{ha} \text { ) }\end{array}$ \\
\hline Spioncello & 30 territori per $\mathrm{km}^{2}$ (più elevata in aree particolarmente idonee) \\
\hline Cutrettola & $\begin{array}{l}\text { a scala locale: } 1 \text { coppia per } 10 \text { ha } \\
\text { a scala di comprensorio: } 5 \text { coppie per } \mathrm{km}^{2}\end{array}$ \\
\hline Ballerina gialla & $\begin{array}{l}\text { a scala locale: } 3 \text { coppie per km di corso d'acqua } \\
\text { a scala di comprensorio: } 1 \text { coppia per km di corso d'acqua per aree vocate, } 0.1 \text { coppie per } \\
\text { km per aree meno idonee }\end{array}$ \\
\hline Ballerina bianca & $\begin{array}{l}\text { a scala locale: } 1.5 \text { coppie per } 10 \text { ha, } 4.5 \text { coppie per } 10 \text { ha in contesti particolarmente } \\
\text { ottimali } \\
\text { a scala di comprensorio: } 4 \text { coppie per } \mathrm{km}^{2}\end{array}$ \\
\hline Merlo acquaiolo & $\begin{array}{l}\text { in ambienti ottimali, a scala locale: } 2 \text { coppie per } \mathrm{km} \text { di corso d'acqua; } 1 \text { coppia per } \mathrm{km} \text { in } \\
\text { Sicilia e Appennino centrale e meridionale (vedi testo) }\end{array}$ \\
\hline Scricciolo & $\begin{array}{l}\text { a scala locale: } 23-25 \text { coppie per } 10 \text { ha in ambienti idonei; } 5-8 \text { coppie per } 10 \text { ha in ambienti } \\
\text { sub-ottimali planiziali }\end{array}$ \\
\hline Passera scopaiola & a scala locale: $3-5$ coppie riproduttive per 10 ha. \\
\hline Sordone & 5-6 territori per $\mathrm{km}^{2}$ (vedi testo) \\
\hline Pettirosso & $\begin{array}{l}\text { a scala locale: } 6 \text { coppie per } 10 \text { ha per ambienti idonei, } 14 \text { coppie per } 10 \text { ha in contesti } \\
\text { particolarmente vocati, } \\
\text { a scala di comprensorio: } 10 \text { territori per } \mathrm{km}^{2} \text { (aree mediamente idonee di diversi } \mathrm{km}^{2} \mathrm{di} \\
\text { estensione) }\end{array}$ \\
\hline
\end{tabular}




\begin{tabular}{|c|c|}
\hline Specie & FRV \\
\hline Usignolo & $\begin{array}{l}\text { a scala locale: } 19 \text { coppie per } 10 \text { ha a in ambienti particolarmente vocati; in contesti sub- } \\
\text { ottimali } 5 \text { coppie per } 10 \text { ha }\end{array}$ \\
\hline Codirosso spazzacamino & $\begin{array}{l}\text { a scala locale: } 6 \text { coppie per } 10 \text { ha (in aree particolarmente idonee questo valore può essere } \\
\text { superato; in ambito urbano valore provvisorio di } 1.2 \text { coppie per } 10 \text { ha) }\end{array}$ \\
\hline Codirosso comune & $\begin{array}{l}\text { a scala locale: } 5 \text { coppie per } 10 \text { ha ( } 8 \text { coppie per } 10 \text { ha per ambienti o contesti particolarmente } \\
\text { idonei) }\end{array}$ \\
\hline Stiaccino & a scala locale: 7 coppie per 10 ha \\
\hline Saltimpalo & $\begin{array}{l}\text { a scala locale: } 5 \text { coppie per } 10 \text { ha } \\
\text { a scala di comprensorio: } 15 \text { coppie per } \mathrm{km}^{2}\end{array}$ \\
\hline Culbianco & $\begin{array}{l}\text { a scala locale: } 5 \text { coppie per } 10 \text { ha } \\
\text { scala di comprensorio (provvisorio): } 11 \text { coppie per } \mathrm{km}^{2}\end{array}$ \\
\hline Codirossone & a scala locale: 4 coppie per 10 ha (vedi testo) \\
\hline Passero solitario & $\begin{array}{l}\text { a scala locale: } 3 \text { coppie per } 10 \text { ha } \\
\text { a scala di comprensorio: } 15 \text { coppie per } \mathrm{km}^{2} \\
\text { falesie rocciose con esteso sviluppo lineare: } 2.5 \text { maschi per km }\end{array}$ \\
\hline Merlo & $\begin{array}{l}\text { a scala locale: } 10 \text { coppie per } 10 \text { ha; in contesti ottimali (suburbani) } 36 \text { coppie per } 10 \text { ha; in } \\
\text { contesti agricoli intensivamente coltivati } 1 \text { coppia per } 10 \text { ha. }\end{array}$ \\
\hline Merlo dal collare & $\begin{array}{l}\text { a scala locale: } 1.7 \text { coppie per } 10 \text { ha } \\
\text { a scala di comprensorio: } 3 \text { coppie per } \mathrm{km}^{2}\end{array}$ \\
\hline Tordo bottaccio & a scala locale: 2 coppie per 10 ha, 5 coppie per 10 ha in contesti ottimali \\
\hline Tordela & a scala locale: 3 coppie per 10 ha \\
\hline Usignolo di fiume & $\begin{array}{l}\text { a scala locale: } 10 \text { coppie per } 10 \text { ha (annate favorevoli) } \\
\text { a scala di comprensorio, lungo corsi d'acqua: } 5 \text { coppie per } \mathrm{km} \text { in ambienti favorevoli, } 15 \\
\text { coppie per km in ambienti particolarmente idonei, } 1 \text { coppia per km per aree meno vocate }\end{array}$ \\
\hline Beccamoschino & a scala locale: 10 coppie per 10 ha (annate favorevoli) \\
\hline Cannaiola verdognola & $\begin{array}{l}\text { a scala locale: } 10 \text { coppie per ha per ambienti particolarmente idonei e } 10 \text { coppie per } 10 \text { ha } \\
\text { per ambienti mediamente vocati }\end{array}$ \\
\hline Cannaiola comune & $\begin{array}{l}\text { a scala locale: } 5 \text { coppie per ha di canneto; in ambienti particolarmente favorevoli } 15 \text { coppie } \\
\text { per ha di canneto }\end{array}$ \\
\hline Cannareccione & a scala locale: 10 coppie per 10 ha di canneto \\
\hline Canapino comune & a scala locale: 1 coppia per ha \\
\hline Sterpazzola della Sardegna & $\begin{array}{l}\text { a scala locale: } 7 \text { coppie per } 10 \text { ha per ambienti particolarmente vocati; } 5 \text { coppie per } 10 \text { ha } \\
\text { per altri ambienti idonei alla specie }\end{array}$ \\
\hline Sterpazzolina comune & a scala locale: 6 coppie per 10 ha (localmente superiore; vedi testo) \\
\hline Sterpazzolina di Moltoni & $\begin{array}{l}\text { a scala locale: } 10 \text { coppie per } 10 \text { ha } \\
\text { a scala di comprensorio: } 50 \text { coppie per } \mathrm{km}^{2} \text { (vedi testo) }\end{array}$ \\
\hline Occhiocotto & $\begin{array}{l}\text { a scala locale: } 15 \text { coppie per } 10 \text { ha per macchia costiera; } 5 \text { coppie per } 10 \text { ha in ambienti } \\
\text { dell'entroterra }\end{array}$ \\
\hline Bigiarella & a scala locale: 4 coppie per 10 ha (vedi testo) \\
\hline Sterpazzola & a scala locale: 7 coppie per 10 ha (localmente superabile) \\
\hline Beccafico & $\begin{array}{l}\text { a scala locale: } 5 \text { coppie per } 10 \text { ha } \\
\text { scala di comprensorio: } 25 \text { coppie per } \mathrm{km}^{2}\end{array}$ \\
\hline Capinera & $\begin{array}{l}\text { a scala locale: } 10 \text { coppie per } 10 \text { ha per ambienti di qualche decina di ettari; per tessere di } \\
\text { habitat di piccole dimensioni e particolarmente idonee: } 30 \text { coppie per } 10 \text { ha (valore che } \\
\text { localmente può essere comunque superato) } \\
\text { a scala di comprensorio: } 70 \text { coppie per } \mathrm{km}^{2}\end{array}$ \\
\hline Luì bianco & $\begin{array}{l}\text { a scala locale: } 10 \text { coppie per } 10 \text { ha per habitat ottimali e } 5 \text { coppie per } 10 \text { ha per altri ambienti } \\
\text { idonei alla specie }\end{array}$ \\
\hline Luì verde & a scala locale: 3 coppie per 10 ha \\
\hline
\end{tabular}




\begin{tabular}{|c|c|}
\hline Specie & FRV \\
\hline Luì piccolo & $\begin{array}{l}\text { a scala locale: } 5 \text { coppie per } 10 \text { ha } \\
\text { a scala di comprensorio: } 15 \text { coppie per } \mathrm{km}^{2}\end{array}$ \\
\hline Regolo & a scala locale: 12 coppie per 10 ha \\
\hline Fiorrancino & a scala locale: 10 coppie per 10 ha \\
\hline Pigliamosche & $\begin{array}{l}\text { a scala locale: } 5 \text { coppie per } 10 \text { ha; } 10 \text { coppie per } 10 \text { ha per habitat particolarmente idonei, } \\
2 \text { coppie per } 10 \text { in siti sub-ottimali }\end{array}$ \\
\hline Codibugnolo & a scala locale: 4 coppie per 10 ha \\
\hline Cinciarella & a scala locale: 10 coppie per 10 ha; 3 coppie per 10 ha per ambienti sub-ottimali \\
\hline Cinciallegra & a scala locale: 15 coppie per 10 ha, 2 coppie per 10 ha in habitat sub-ottimali \\
\hline Cincia dal ciuffo & $\begin{array}{l}\text { a scala locale: } 2.5 \text { coppie per } 10 \text { ha; in aree planiziali o basso collinari: } 1.5 \text { coppie per } 10 \\
\text { ha }\end{array}$ \\
\hline Cincia mora & a scala locale: 5 coppie per 10 ha, 2 coppie per 10 ha in boschi planiziali e collinari \\
\hline Cincia alpestre & a scala locale: 3 coppie per 10 ha \\
\hline Cincia bigia & a scala locale: 5 coppie per 10 ha \\
\hline Picchio muratore & $\begin{array}{l}\text { a scala locale: } 5 \text { coppie per } 10 \text { ha per fustaie mature, } 1 \text { coppia per } 10 \text { ha per boschi non } \\
\text { ottimali }\end{array}$ \\
\hline Rampichino alpestre & a scala locale: 3 coppie per 10 ha \\
\hline Rampichino comune & a scala locale: 5 coppie per 10 ha \\
\hline Rigogolo & a scala locale: 3 coppie per 10 ha \\
\hline Averla capirossa & sottospecie nominale, Italia continentale: a scala di comprensorio: 5 coppie per $\mathrm{km}^{2}$ \\
\hline Nocciolaia & a scala di comprensorio: 10 coppie per $\mathrm{km}^{2}$ \\
\hline Passera europea & provvisoriamente utilizzabili i dati relativi alla passera d'Italia \\
\hline Passera d'Italia & $\begin{array}{l}\text { per situazioni non coloniali: } \\
\text { a scala locale: } 1,2 \text { coppie per } 10 \mathrm{ha} \\
\text { a scala di comprensorio: } 200 \text { coppie per } \mathrm{km}^{2}\end{array}$ \\
\hline Fringuello & a scala locale: 5 coppie per 10 ha \\
\hline Verdone & $\begin{array}{l}\text { a scala locale: } 5 \text { coppie per } 10 \text { ha in ambienti urbani e suburbani, } 1 \text { coppia per } 10 \text { ha in } \\
\text { ambienti rurali; } \\
\text { a scala di comprensorio: } 10 \text { coppie per } \mathrm{km}^{2} \text { in ambienti urbani e suburbani e di } 6 \text { coppie per } \\
\mathrm{km}^{2} \text { in ambienti rurali (vedi testo) }\end{array}$ \\
\hline Cardellino & $\begin{array}{l}\text { a scala locale: } 10 \text { coppie per } 10 \text { ha, } 3 \text { coppie per ha per piccoli ambienti particolarmente } \\
\text { idonei; } \\
\text { a scala di comprensorio: } 15 \text { coppie per } \mathrm{km}^{2}\end{array}$ \\
\hline Fanello & $\begin{array}{l}\text { a scala locale: } 10 \text { coppie per } 10 \text { ha per l'Italia continentale, } 50 \text { coppie per } 10 \text { ha per la } \\
\text { Sicilia (localmente superabile) }\end{array}$ \\
\hline Organetto & a scala locale: 3 coppie per ha \\
\hline Zigolo giallo & a scala locale: 3 coppie per 10 ha \\
\hline Zigolo nero & $\begin{array}{l}\text { a scala locale: } 5 \text { coppie per } 10 \text { ha } \\
\text { a scala di comprensorio: } 20 \text { coppie per } \mathrm{km}^{2}\end{array}$ \\
\hline Zigolo muciatto & a scala locale: 3 coppie per 10 ha \\
\hline Migliarino di palude & a scala locale: 5 coppie per 10 ha \\
\hline Zigolo capinero & $\begin{array}{l}\text { a scala locale: } 6 \text { coppie per } 10 \text { ha } \\
\text { a scala di comprensorio: } 10 \text { coppie per } \mathrm{km}^{2} \text { (vedi testo) }\end{array}$ \\
\hline Strillozzo & $\begin{array}{l}\text { a scala locale: } 5 \text { maschi cantori per } 10 \text { ha } \\
\text { a scala di comprensorio: } 30 \text { maschi cantori per } \mathrm{km}^{2} \text { (vedi testo) }\end{array}$ \\
\hline
\end{tabular}


Tab. 3 - Classificazione "a semaforo" per range, popolazione, habitat e stato di conservazione complessivo per le specie inserite nell'Allegato I della Direttiva Uccelli (Gustin et al., 2009). Classificazione rivista dopo Nardelli et al. (2015). / "Traffic light" classification for range, population, habitat and overall conservation status for species included in Annex I of Birds Directive (Gustin et al., 2009). Classification reviewed after Nardelli et al. (2015).

\begin{tabular}{|c|c|c|c|c|}
\hline Specie & Range & Popolazione & Habitat & Complessivo \\
\hline Berta minore & inadeguato & sconosciuto & cattivo & cattivo \\
\hline Berta maggiore & favorevole & favorevole & cattivo & cattivo \\
\hline Uccello delle tempeste & sconosciuto & sconosciuto & inadeguato & inadeguato \\
\hline Marangone dal ciuffo & favorevole & inadeguato & favorevole & inadeguato \\
\hline Marangone minore & favorevole & inadeguato & favorevole & inadeguato \\
\hline Tarabuso & inadeguato & cattivo & inadeguato & cattivo \\
\hline Tarabusino & inadeguato & cattivo & inadeguato & cattivo \\
\hline Nitticora & favorevole & cattivo & inadeguato & cattivo \\
\hline Sgarza ciuffetto & favorevole & inadeguato & favorevole & inadeguato \\
\hline Garzetta & favorevole & inadeguato & favorevole & inadeguato \\
\hline Airone bianco maggiore & favorevole & inadeguato & favorevole & inadeguato \\
\hline Airone rosso & favorevole & favorevole & favorevole & favorevole \\
\hline Cicogna nera & favorevole & cattivo & favorevole & cattivo \\
\hline Cicogna bianca & favorevole & inadeguato & favorevole & inadeguato \\
\hline Mignattaio & cattivo & cattivo & inadeguato & cattivo \\
\hline Spatola & inadeguato & inadeguato & favorevole & inadeguato \\
\hline Fenicottero & inadeguato & inadeguato & inadeguato & inadeguato \\
\hline Anatra marmorizzata & cattivo & cattivo & inadeguato & cattivo \\
\hline Moretta tabaccata & inadeguato & sconosciuto & inadeguato & inadeguato \\
\hline Falco pecchiaiolo & favorevole & sconosciuto & favorevole & favorevole \\
\hline Nibbio bruno & favorevole & inadeguato & inadeguato & inadeguato \\
\hline Nibbio reale & inadeguato & cattivo & inadeguato & cattivo \\
\hline Gipeto & cattivo & cattivo & favorevole & cattivo \\
\hline Capovaccaio & cattivo & cattivo & cattivo & cattivo \\
\hline Grifone & cattivo & inadeguato & inadeguato & cattivo \\
\hline Biancone & favorevole & favorevole & inadeguato & inadeguato \\
\hline Falco di palude & favorevole & sconosciuto & favorevole & favorevole \\
\hline Albanella minore & favorevole & cattivo & inadeguato & cattivo \\
\hline Astore di Sardegna & sconosciuto & cattivo & inadeguato & cattivo \\
\hline Aquila reale & favorevole & favorevole & inadeguato & inadeguato \\
\hline Aquila di Bonelli & cattivo & cattivo & sconosciuto & cattivo \\
\hline Grillaio & favorevole & favorevole & cattivo & cattivo \\
\hline Falco della regina & inadeguato & cattivo & favorevole & cattivo \\
\hline Falco cuculo & inadeguato & cattivo & inadeguato & cattivo \\
\hline Lanario & inadeguato & cattivo & cattivo & cattivo \\
\hline Falco pellegrino & favorevole & favorevole & favorevole & favorevole \\
\hline Francolino di monte & inadeguato & sconosciuto & inadeguato & inadeguato \\
\hline Pernice bianca & cattivo & cattivo & cattivo & cattivo \\
\hline
\end{tabular}




\begin{tabular}{|c|c|c|c|c|}
\hline Specie & Range & Popolazione & Habitat & Complessivo \\
\hline Fagiano di monte & favorevole & cattivo & inadeguato & cattivo \\
\hline Gallo cedrone & cattivo & cattivo & inadeguato & cattivo \\
\hline Coturnice & cattivo & cattivo & cattivo & cattivo \\
\hline Coturnice di Sicilia & cattivo & cattivo & inadeguato & cattivo \\
\hline Pernice sarda & inadeguato & inadeguato & favorevole & inadeguato \\
\hline Voltolino & cattivo & cattivo & inadeguato & cattivo \\
\hline Schiribilla & inadeguato & cattivo & inadeguato & cattivo \\
\hline Re di quaglie & inadeguato & cattivo & cattivo & cattivo \\
\hline Pollo sultano & favorevole & favorevole & inadeguato & inadeguato \\
\hline Gallina prataiola & cattivo & cattivo & cattivo & cattivo \\
\hline Cavaliere d'Italia & favorevole & sconosciuto & favorevole & favorevole \\
\hline Avocetta & favorevole & sconosciuto & inadeguato & inadeguato \\
\hline Occhione & inadeguato & favorevole & inadeguato & inadeguato \\
\hline Pernice di mare & inadeguato & sconosciuto & inadeguato & inadeguato \\
\hline Fratino & inadeguato & cattivo & cattivo & cattivo \\
\hline Piviere tortolino & cattivo & cattivo & inadeguato & cattivo \\
\hline Gabbiano corallino & inadeguato & inadeguato & inadeguato & inadeguato \\
\hline Gabbiano roseo & favorevole & inadeguato & inadeguato & inadeguato \\
\hline Gabbiano corso & favorevole & inadeguato & inadeguato & inadeguato \\
\hline Sterna zampenere & inadeguato & sconosciuto & sconosciuto & inadeguato \\
\hline Beccapesci & inadeguato & sconosciuto & inadeguato & inadeguato \\
\hline Sterna comune & inadeguato & inadeguato & inadeguato & inadeguato \\
\hline Fraticello & inadeguato & cattivo & inadeguato & cattivo \\
\hline Mignattino piombato & inadeguato & sconosciuto & inadeguato & inadeguato \\
\hline Mignattino comune & cattivo & cattivo & inadeguato & cattivo \\
\hline Gufo reale & inadeguato & inadeguato & inadeguato & inadeguato \\
\hline Civetta nana & inadeguato & inadeguato & inadeguato & inadeguato \\
\hline Allocco degli Urali & inadeguato & inadeguato & favorevole & inadeguato \\
\hline Civetta capogrosso & inadeguato & inadeguato & inadeguato & inadeguato \\
\hline Succiacapre & inadeguato & sconosciuto & inadeguato & inadeguato \\
\hline Martin pescatore & favorevole & inadeguato & inadeguato & inadeguato \\
\hline Ghiandaia marina & inadeguato & inadeguato & sconosciuto & inadeguato \\
\hline Picchio cenerino & sconosciuto & favorevole & favorevole & favorevole \\
\hline Picchio nero & favorevole & inadeguato & favorevole & inadeguato \\
\hline Picchio rosso mezzano & inadeguato & inadeguato & favorevole & inadeguato \\
\hline Picchio dalmatino & inadeguato & inadeguato & sconosciuto & inadeguato \\
\hline Picchio tridattilo & favorevole & inadeguato & favorevole & inadeguato \\
\hline Calandra & cattivo & cattivo & cattivo & cattivo \\
\hline Calandrella & cattivo & cattivo & cattivo & cattivo \\
\hline Tottavilla & inadeguato & inadeguato & inadeguato & inadeguato \\
\hline Calandro & inadeguato & cattivo & inadeguato & cattivo \\
\hline Forapaglie castagnolo & cattivo & cattivo & cattivo & cattivo \\
\hline
\end{tabular}




\begin{tabular}{|l|c|c|c|c|}
\hline Specie & Range & Popolazione & Habitat & Complessivo \\
\hline Magnanina sarda & favorevole & sconosciuto & sconosciuto & sconosciuto \\
\hline Magnanina comune & favorevole & sconosciuto & inadeguato & inadeguato \\
\hline Bigia padovana & cattivo & cattivo & inadeguato & cattivo \\
\hline Balia dal collare & cattivo & cattivo & inadeguato & cattivo \\
\hline Averla piccola & inadeguato & cattivo & cattivo & cattivo \\
\hline Averla cenerina & cattivo & cattivo & cattivo & cattivo \\
\hline Gracchio corallino & cattivo & cattivo & inadeguato & cattivo \\
\hline Ortolano & cattivo & sconosciuto & cattivo & cattivo \\
\hline
\end{tabular}

Tab. 4 - Classificazione "a semaforo" per range, popolazione, habitat e stato di conservazione complessivo per le specie non inserite nell'Allegato I della Direttiva Uccelli (Gustin et al., 2010a,b). / "Traffic light" classification for range, population habitat and overall conservation status for species not included in Annex I of Birds Directive (Gustin et al. 2010a,b).

\begin{tabular}{|c|c|c|c|c|}
\hline Specie & Range & Popolazione & Habitat & Complessivo \\
\hline Cigno reale & favorevole & favorevole & favorevole & favorevole \\
\hline Volpoca & favorevole & inadeguato & favorevole & inadeguato \\
\hline Canapiglia & sconosciuto & sconosciuto & favorevole & sconosciuto \\
\hline Alzavola & inadeguato & sconosciuto & favorevole & inadeguato \\
\hline Germano reale & favorevole & favorevole & favorevole & favorevole \\
\hline Marzaiola & inadeguato & sconosciuto & cattivo & cattivo \\
\hline Mestolone & inadeguato & sconosciuto & favorevole & inadeguato \\
\hline Fistione turco & inadeguato & inadeguato & favorevole & inadeguato \\
\hline Moriglione & inadeguato & cattivo & favorevole & cattivo \\
\hline Moretta & inadeguato & inadeguato & favorevole & inadeguato \\
\hline Smergo maggiore & favorevole & inadeguata & favorevole & inadeguato \\
\hline Pernice rossa & inadeguato & cattivo & inadeguato & cattivo \\
\hline Starna & cattivo & cattivo & inadeguato & cattivo \\
\hline Quaglia & favorevole & cattivo & inadeguato & cattivo \\
\hline Svasso maggiore & favorevole & sconosciuto & favorevole & favorevole \\
\hline Tuffetto & favorevole & sconosciuto & favorevole & favorevole \\
\hline Cormorano & favorevole & favorevole & favorevole & favorevole \\
\hline Airone guardabuoi & favorevole & favorevole & favorevole & favorevole \\
\hline Airone cenerino & favorevole & inadeguato & favorevole & inadeguato \\
\hline Astore & favorevole & inadeguato & favorevole & inadeguato \\
\hline Sparviere & favorevole & favorevole & favorevole & favorevole \\
\hline Poiana & favorevole & favorevole & favorevole & favorevole \\
\hline Gheppio & favorevole & favorevole & favorevole & favorevole \\
\hline Lodolaio & favorevole & inadeguato & favorevole & inadeguato \\
\hline Porciglione & sconosciuto & sconosciuto & favorevole & sconosciuto \\
\hline Gallinella d'acqua & favorevole & favorevole & favorevole & favorevole \\
\hline Folaga & favorevole & favorevole & favorevole & favorevole \\
\hline Beccaccia di mare & favorevole & inadeguato & inadeguato & inadeguato \\
\hline
\end{tabular}




\begin{tabular}{|c|c|c|c|c|}
\hline Specie & Range & Popolazione & Habitat & Complessivo \\
\hline Corriere piccolo & inadeguato & sconosciuto & inadeguato & inadeguato \\
\hline Pavoncella & favorevole & favorevole & inadeguato & inadeguato \\
\hline Beccaccia & sconosciuto & sconosciuto & sconosciuto & sconosciuto \\
\hline Pittima reale & inadeguato & cattivo & inadeguato & cattivo \\
\hline Piro-piro piccolo & inadeguato & cattivo & inadeguato & cattivo \\
\hline Pettegola & favorevole & inadeguato & inadeguato & inadeguato \\
\hline Gabbiano comune & sconosciuto & sconosciuto & favorevole & sconosciuto \\
\hline Gabbiano reale & favorevole & favorevole & favorevole & favorevole \\
\hline Mignattino alibianche & inadeguato & cattivo & cattivo & cattivo \\
\hline Piccione selvatico & sconosciuto & sconosciuto & favorevole & sconosciuto \\
\hline Colombella & sconosciuto & sconosciuto & sconosciuto & sconosciuto \\
\hline Colombaccio & favorevole & favorevole & favorevole & favorevole \\
\hline Tortora dal collare & favorevole & favorevole & favorevole & favorevole \\
\hline Tortora selvatica & favorevole & sconosciuto & inadeguato & inadeguato \\
\hline Tortora delle palme & inadeguato & inadeguato & favorevole & inadeguato \\
\hline Cuculo dal ciuffo & favorevole & inadeguato & sconosciuto & inadeguato \\
\hline Cuculo & favorevole & inadeguato & favorevole & inadeguato \\
\hline Barbagianni & inadeguato & cattivo & inadeguato & cattivo \\
\hline Assiolo & inadeguato & sconosciuto & inadeguato & inadeguato \\
\hline Civetta & favorevole & sconosciuto & favorevole & favorevole \\
\hline Allocco & favorevole & favorevole & favorevole & favorevole \\
\hline Gufo comune & favorevole & favorevole & favorevole & favorevole \\
\hline Rondone comune & favorevole & inadeguato & inadeguato & inadeguato \\
\hline Rondone maggiore & favorevole & favorevole & favorevole & favorevole \\
\hline Rondone pallido & favorevole & favorevole & favorevole & favorevole \\
\hline Gruccione & favorevole & favorevole & favorevole & favorevole \\
\hline Upupa & favorevole & Sconosciuto & sconosciuto & sconosciuto \\
\hline Torcicollo & inadeguato & cattivo & inadeguato & cattivo \\
\hline Picchio verde & favorevole & favorevole & favorevole & favorevole \\
\hline Picchio rosso maggiore & favorevole & favorevole & favorevole & favorevole \\
\hline Picchio rosso minore & favorevole & favorevole & favorevole & favorevole \\
\hline Cappellaccia & inadeguato & inadeguato & sconosciuto & inadeguato \\
\hline Allodola & inadeguato & cattivo & cattivo & cattivo \\
\hline Topino & cattivo & cattivo & cattivo & cattivo \\
\hline Rondine montana & favorevole & favorevole & favorevole & favorevole \\
\hline Rondine & favorevole & cattivo & inadeguato & cattivo \\
\hline Balestruccio & favorevole & cattivo & inadeguato & cattivo \\
\hline Rondine rossiccia & inadeguato & cattivo & favorevole & cattivo \\
\hline Prispolone & favorevole & inadeguato & inadeguato & inadeguato \\
\hline Spioncello & inadeguato & inadeguato & inadeguato & inadeguato \\
\hline Cutrettola & favorevole & cattivo & inadeguato & cattivo \\
\hline
\end{tabular}




\begin{tabular}{|c|c|c|c|c|}
\hline Specie & Range & Popolazione & Habitat & Complessivo \\
\hline Ballerina gialla & favorevole & favorevole & favorevole & favorevole \\
\hline Ballerina bianca & inadeguato & inadeguato & inadeguato & inadeguato \\
\hline Merlo acquaiolo & favorevole & inadeguato & inadeguato & inadeguato \\
\hline Scricciolo & favorevole & favorevole & favorevole & favorevole \\
\hline Passera scopaiola & favorevole & favorevole & favorevole & favorevole \\
\hline Sordone & inadeguato & inadeguato & inadeguato & inadeguato \\
\hline Pettirosso & favorevole & favorevole & favorevole & favorevole \\
\hline Usignolo & favorevole & favorevole & favorevole & favorevole \\
\hline Codirosso spazzacamino & favorevole & favorevole & favorevole & favorevole \\
\hline Codirosso comune & favorevole & favorevole & favorevole & favorevole \\
\hline Stiaccino & inadeguato & cattivo & inadeguato & cattivo \\
\hline Saltimpalo & favorevole & cattivo & inadeguato & cattivo \\
\hline Culbianco & favorevole & inadeguato & inadeguato & inadeguato \\
\hline Monachella & inadeguato & cattivo & inadeguato & cattivo \\
\hline Codirossone & cattivo & cattivo & cattivo & cattivo \\
\hline Passero solitario & favorevole & sconosciuto & inadeguato & inadeguato \\
\hline Merlo & favorevole & favorevole & favorevole & favorevole \\
\hline Merlo dal collare & favorevole & inadeguato & inadeguato & inadeguato \\
\hline Cesena & inadeguato & cattivo & favorevole & cattivo \\
\hline Tordo bottaccio & favorevole & favorevole & favorevole & favorevole \\
\hline Tordela & inadeguato & inadeguato & favorevole & inadeguato \\
\hline Usignolo di fiume & favorevole & favorevole & favorevole & favorevole \\
\hline Beccamoschino & favorevole & inadeguato & favorevole & inadeguato \\
\hline Salciaiola & cattivo & cattivo & inadeguato & cattivo \\
\hline Forapaglie comune & cattivo & cattivo & cattivo & cattivo \\
\hline Cannaiola verdognola & favorevole & inadeguato & inadeguato & inadeguato \\
\hline Cannaiola comune & favorevole & inadeguato & inadeguato & inadeguato \\
\hline Cannareccione & favorevole & cattivo & inadeguato & cattivo \\
\hline Canapino comune & favorevole & favorevole & favorevole & favorevole \\
\hline Capinera & favorevole & favorevole & favorevole & favorevole \\
\hline Beccafico & inadeguato & cattivo & favorevole & cattivo \\
\hline Bigiarella & inadeguato & sconosciuto & favorevole & inadeguato \\
\hline Bigia grossa & cattivo & cattivo & inadeguato & cattivo \\
\hline Sterpazzola & inadeguato & inadeguato & inadeguato & inadeguato \\
\hline Sterpazzola della Sardegna & sconosciuto & sconosciuto & sconosciuto & sconosciuto \\
\hline Sterpazzolina comune & favorevole & sconosciuto & favorevole & favorevole \\
\hline Sterpazzolina di Moltoni & favorevole & favorevole & favorevole & favorevole \\
\hline Occhiocotto & favorevole & favorevole & favorevole & favorevole \\
\hline Luì bianco & favorevole & favorevole & favorevole & favorevole \\
\hline Luì verde & favorevole & inadeguato & favorevole & inadeguato \\
\hline Luì piccolo & favorevole & favorevole & favorevole & favorevole \\
\hline
\end{tabular}




\begin{tabular}{|c|c|c|c|c|}
\hline Specie & Range & Popolazione & Habitat & Complessivo \\
\hline Regolo & favorevole & inadeguato & favorevole & inadeguato \\
\hline Fiorrancino & favorevole & favorevole & favorevole & favorevole \\
\hline Pigliamosche & favorevole & favorevole & favorevole & favorevole \\
\hline Basettino & cattivo & cattivo & inadeguato & cattivo \\
\hline Codibugnolo & favorevole & favorevole & favorevole & favorevole \\
\hline Cinciarella & favorevole & favorevole & favorevole & favorevole \\
\hline Cinciallegra & favorevole & favorevole & favorevole & favorevole \\
\hline Cincia dal ciuffo & favorevole & favorevole & favorevole & favorevole \\
\hline Cincia mora & favorevole & favorevole & favorevole & favorevole \\
\hline Cincia alpestre & favorevole & favorevole & favorevole & favorevole \\
\hline Cincia bigia & favorevole & favorevole & favorevole & favorevole \\
\hline Picchio muratore & favorevole & favorevole & favorevole & favorevole \\
\hline Picchio muraiolo & favorevole & sconosciuto & favorevole & favorevole \\
\hline Rampichino alpestre & favorevole & favorevole & favorevole & favorevole \\
\hline Rampichino comune & favorevole & favorevole & favorevole & favorevole \\
\hline Pendolino & cattivo & cattivo & sconosciuto & cattivo \\
\hline Rigogolo & favorevole & favorevole & favorevole & favorevole \\
\hline Averla capirossa & cattivo & cattivo & cattivo & cattivo \\
\hline Ghiandaia & favorevole & favorevole & favorevole & favorevole \\
\hline Gazza & favorevole & favorevole & favorevole & favorevole \\
\hline Nocciolaia & favorevole & favorevole & favorevole & favorevole \\
\hline Gracchio alpino & favorevole & inadeguato & favorevole & inadeguato \\
\hline Taccola & favorevole & favorevole & favorevole & favorevole \\
\hline Cornacchia nera & favorevole & favorevole & favorevole & favorevole \\
\hline Cornacchia grigia & favorevole & favorevole & favorevole & favorevole \\
\hline Corvo imperiale & favorevole & favorevole & favorevole & favorevole \\
\hline Storno nero & favorevole & sconosciuto & favorevole & favorevole \\
\hline Storno & favorevole & favorevole & favorevole & favorevole \\
\hline Passera europea & favorevole & sconosciuto & sconosciuto & sconosciuto \\
\hline Passera d'Italia & favorevole & cattivo & inadeguato & cattivo \\
\hline Passera sarda & sconosciuto & sconosciuto & inadeguato & sconosciuto \\
\hline Passera mattugia & favorevole & cattivo & inadeguato & cattivo \\
\hline Passera lagia & inadeguato & cattivo & inadeguato & cattivo \\
\hline Fringuello alpino & cattivo & sconosciuto & sconosciuto & cattivo \\
\hline Fringuello & favorevole & favorevole & favorevole & favorevole \\
\hline Verzellino & favorevole & favorevole & favorevole & favorevole \\
\hline Verdone & favorevole & inadeguato & favorevole & inadeguato \\
\hline Cardellino & favorevole & inadeguato & favorevole & inadeguato \\
\hline Venturone alpino & favorevole & sconosciuto & sconosciuto & sconosciuto \\
\hline Venturone corso & favorevole & sconosciuto & sconosciuto & sconosciuto \\
\hline Lucherino & inadeguato & sconosciuto & sconosciuto & sconosciuto \\
\hline
\end{tabular}




\begin{tabular}{|l|c|c|c|c|}
\hline Specie & Range & Popolazione & Habitat & Complessivo \\
\hline Fanello & favorevole & inadeguato & inadeguato & inadeguato \\
\hline Organetto & favorevole & sconosciuto & sconosciuto & sconosciuto \\
\hline Crociere & favorevole & favorevole & favorevole & favorevole \\
\hline Ciuffolotto & favorevole & inadeguato & sconosciuto & inadeguato \\
\hline Frosone & inadeguato & sconosciuto & inadeguato & inadeguato \\
\hline Zigolo giallo & inadeguato & cattivo & cattivo & cattivo \\
\hline Zigolo nero & favorevole & favorevole & inadeguato & inadeguato \\
\hline Zigolo muciatto & favorevole & sconosciuto & sconosciuto & sconosciuto \\
\hline Migliarino di palude & cattivo & cattivo & cattivo & cattivo \\
\hline Zigolo capinero & sconosciuto & sconosciuto & sconosciuto & sconosciuto \\
\hline Strillozzo & inadeguato & favorevole & inadeguato & inadeguato \\
\hline
\end{tabular}

Per alcune specie, la valutazione dello stato di conservazione appare differente all' interno delle diverse regioni biogeografiche: alpina, continentale e mediterranea (Tab. 5).

Considerando i diversi Ordini, tra quelli che contano almeno 5 specie nidificanti, la situazione più preoccupante (con oltre il 40\% di specie con stato di conservazione cattivo) è rappresentata da Galliformi, Accipitriformi e Gruiformi, mentre all'opposto Columbiformi, Strigiformi e Piciformi mostrano le percentuali più basse di specie con stato di conservazione cattivo (Tab. 6).

Complessivamente, 42 specie incluse nell'Allegato I della Direttiva Uccelli hanno stato di conservazione cattivo, 39 inadeguato, 6 favorevole e 1 sconosciuto; tra le specie non inserite nell'allegato I, 35 hanno stato di conservazione cattivo, 44 inadeguato, 67 favorevole e 16 sconosciuto (Fig. 1). Da questi dati si evince lo stato di conservazione mediamente più sfavorevole delle specie in Allegato I rispetto a quelle non in Allegato, che mostrano complessivamente uno stato di conservazione favorevole con maggior frequenza. Lo stato di conservazione appare mediamente peggiore nella bioregione continentale rispetto a quelle alpina e mediterranea (Fig. 2).

Considerando le diverse macro tipologie ambientali, boschi e foreste presentano la situazione più favorevole, mentre i mosaici agricoli rappresentano l'ambiente con lo stato di conservazione mediamente più critico (Fig. 3).

Tab. 5 - Classificazione "a semaforo" per alcune popolazioni a scala bioregionale (alpina, continentale e mediterranea). / "Traffic light" classification for some populations at bioregional scale (alpine, continental and mediterranean).

\begin{tabular}{|l|c|c|c|c|}
\hline Specie & Range & Popolazione & Habitat & Complessivo \\
\hline Volpoca (continentale) & favorevole & inadeguato & favorevole & inadeguato \\
\hline Volpoca (mediterranea) & favorevole & cattivo & favorevole & cattivo \\
\hline $\begin{array}{l}\text { Cormorano (continentale } \\
\text { e alpina) }\end{array}$ & favorevole & favorevole & favorevole & favorevole \\
\hline $\begin{array}{l}\text { Cormorano } \\
\text { (mediterranea) }\end{array}$ & favorevole & cattivo & favorevole & cattivo \\
\hline Tarabuso (continentale) & favorevole & inadeguato & cattivo & cattivo \\
\hline Tarabuso (mediterranea) & cattivo & cattivo & cattivo & cattivo \\
\hline Nitticora (continentale) & favorevole & cattivo & inadeguato & cattivo \\
\hline Nitticora (mediterranea) & favorevole & favorevole & sconosciuto & favorevole \\
\hline $\begin{array}{l}\text { Airone rosso } \\
\text { (continentale) }\end{array}$ & favorevole & favorevole & favorevole & favorevole \\
\hline $\begin{array}{l}\text { Airone rosso } \\
\text { (mediterranea) }\end{array}$ & favorevole & inadeguato & inadeguato & inadeguato \\
\hline
\end{tabular}




\begin{tabular}{|c|c|c|c|c|}
\hline Specie & Range & Popolazione & Habitat & Complessivo \\
\hline Aquila reale (alpina) & favorevole & favorevole & inadeguato & inadeguato \\
\hline $\begin{array}{l}\text { Aquila reale } \\
\text { (mediterranea) }\end{array}$ & favorevole & inadeguato & inadeguato & inadeguato \\
\hline $\begin{array}{l}\text { Lodolaio (continentale e } \\
\text { alpina) }\end{array}$ & favorevole & favorevole & favorevole & favorevole \\
\hline Lodolaio (mediterranea) & inadeguato & inadeguato & favorevole & inadeguato \\
\hline Pettegola (continentale) & favorevole & favorevole & inadeguato & inadeguato \\
\hline Pettegola (mediterranea) & favorevole & cattivo & inadeguato & cattivo \\
\hline $\begin{array}{l}\text { Tortora selvatica } \\
\text { (continentale + alpina) }\end{array}$ & inadeguato & cattivo & inadeguato & cattivo \\
\hline $\begin{array}{l}\text { Tortora selvatica } \\
\text { (mediterranea) }\end{array}$ & favorevole & inadeguato & favorevole & inadeguato \\
\hline Gufo reale (alpina) & favorevole & inadeguato & inadeguato & inadeguato \\
\hline $\begin{array}{l}\text { Gufo reale } \\
\text { (mediterranea) }\end{array}$ & cattivo & inadeguato & cattivo & cattivo \\
\hline $\begin{array}{l}\text { Barbagianni } \\
\text { (continentale }+ \text { alpina) }\end{array}$ & cattivo & cattivo & inadeguato & cattivo \\
\hline $\begin{array}{l}\text { Barbagianni } \\
\text { (mediterranea) }\end{array}$ & favorevole & inadeguato & inadeguato & inadeguato \\
\hline $\begin{array}{l}\text { Assiolo (continentale }+ \\
\text { alpina) }\end{array}$ & inadeguato & sconosciuto & inadeguato & inadeguato \\
\hline Assiolo (mediterranea) & favorevole & sconosciuto & favorevole & favorevole \\
\hline $\begin{array}{l}\text { Rondone maggiore } \\
\text { (alpina) }\end{array}$ & inadeguato & inadeguato & sconosciuto & inadeguato \\
\hline $\begin{array}{l}\text { Rondone maggiore } \\
\text { (continentale) }\end{array}$ & favorevole & favorevole & favorevole & favorevole \\
\hline $\begin{array}{l}\text { Rondone maggiore } \\
\text { (mediterranea) }\end{array}$ & favorevole & inadeguato & favorevole & inadeguato \\
\hline Upupa (alpina) & cattivo & cattivo & inadeguato & cattivo \\
\hline Upupa (continentale) & favorevole & inadeguato & inadeguato & inadeguato \\
\hline Upupa (mediterranea) & favorevole & favorevole & sconosciuto & favorevole \\
\hline Picchio nero (alpina) & favorevole & favorevole & favorevole & favorevole \\
\hline $\begin{array}{l}\text { Picchio nero } \\
\text { (mediterranea) }\end{array}$ & inadeguato & inadeguato & favorevole & inadeguato \\
\hline $\begin{array}{l}\text { Torcicollo (alpina e } \\
\text { continentale) }\end{array}$ & inadeguato & cattivo & inadeguato & cattivo \\
\hline $\begin{array}{l}\text { Torcicollo } \\
\text { (mediterranea) }\end{array}$ & favorevole & inadeguato & inadeguato & inadeguato \\
\hline $\begin{array}{l}\text { Cappellaccia } \\
\text { (continentale) }\end{array}$ & cattivo & cattivo & sconosciuto & cattivo \\
\hline
\end{tabular}




\begin{tabular}{|c|c|c|c|c|}
\hline Specie & Range & Popolazione & Habitat & Complessivo \\
\hline $\begin{array}{l}\text { Cappellaccia } \\
\text { (mediterranea) }\end{array}$ & favorevole & favorevole & sconosciuto & favorevole \\
\hline Tottavilla (alpina) & cattivo & cattivo & inadeguato & cattivo \\
\hline $\begin{array}{l}\text { Tottavilla (cont. e } \\
\text { mediterranea) }\end{array}$ & favorevole & favorevole & inadeguato & inadeguato \\
\hline Rondine (continentale) & favorevole & cattivo & inadeguato & cattivo \\
\hline Rondine (mediterranea) & favorevole & favorevole & inadeguato & inadeguato \\
\hline $\begin{array}{l}\text { Spioncello (alpina e } \\
\text { continentale) }\end{array}$ & favorevole & inadeguato & favorevole & inadeguato \\
\hline $\begin{array}{l}\text { Spioncello } \\
\text { (mediterranea) }\end{array}$ & favorevole & inadeguato & inadeguato & inadeguato \\
\hline Ballerina bianca (alpina) & favorevole & favorevole & favorevole & favorevole \\
\hline $\begin{array}{l}\text { Ballerina bianca } \\
\text { (continentale) }\end{array}$ & inadeguato & inadeguato & inadeguato & inadeguato \\
\hline $\begin{array}{l}\text { Ballerina bianca } \\
\text { (mediterranea) }\end{array}$ & favorevole & favorevole & favorevole & favorevole \\
\hline Cutrettola (continentale) & favorevole & inadeguato & inadeguato & inadeguato \\
\hline $\begin{array}{l}\text { Cutrettola } \\
\text { (mediterranea) }\end{array}$ & favorevole & favorevole & favorevole & favorevole \\
\hline Ballerina gialla (alpina) & favorevole & favorevole & favorevole & favorevole \\
\hline $\begin{array}{l}\text { Ballerina gialla } \\
\text { (continentale) }\end{array}$ & inadeguato & inadeguato & sconosciuto & inadeguato \\
\hline $\begin{array}{l}\text { Ballerina gialla } \\
\text { (mediterranea) }\end{array}$ & favorevole & favorevole & favorevole & favorevole \\
\hline $\begin{array}{l}\text { Merlo acquaiolo } \\
\text { (alpino+continentale) }\end{array}$ & favorevole & favorevole & inadeguato & inadeguato \\
\hline $\begin{array}{l}\text { Merlo acquaiolo } \\
\text { (mediterranea) }\end{array}$ & cattivo & cattivo & inadeguato & cattivo \\
\hline Sordone (mediterranea) & favorevole & sconosciuto & favorevole & favorevole \\
\hline Sordone (continentale) & inadeguato & sconosciuto & favorevole & inadeguato \\
\hline $\begin{array}{l}\text { Codirosso comune } \\
\text { (alpina) }\end{array}$ & favorevole & favorevole & favorevole & favorevole \\
\hline $\begin{array}{l}\text { Codirosso comune } \\
\text { (continentale) }\end{array}$ & favorevole & favorevole & favorevole & favorevole \\
\hline $\begin{array}{l}\text { Codirosso comune } \\
\text { (mediterranea) }\end{array}$ & inadeguato & favorevole & favorevole & inadeguato \\
\hline $\begin{array}{l}\text { Merlo dal collare } \\
\text { (continentale) }\end{array}$ & inadeguato & sconosciuto & sconosciuto & sconosciuto \\
\hline $\begin{array}{l}\text { Merlo dal collare } \\
\text { (mediterranea) }\end{array}$ & favorevole & sconosciuto & sconosciuto & sconosciuto \\
\hline
\end{tabular}




\begin{tabular}{|c|c|c|c|c|}
\hline Specie & Range & Popolazione & Habitat & Complessivo \\
\hline $\begin{array}{l}\text { Passero solitario } \\
\text { (alpina }+ \text { continentale) }\end{array}$ & inadeguato & inadeguato & inadeguato & inadeguato \\
\hline $\begin{array}{l}\text { Passero solitario } \\
\text { (mediterranea) }\end{array}$ & favorevole & favorevole & favorevole & favorevole \\
\hline Beccafico (alpina) & favorevole & favorevole & favorevole & favorevole \\
\hline Beccafico (continentale) & cattivo & inadeguato & sconosciuto & cattivo \\
\hline $\begin{array}{l}\text { Beccamoschino (alpina } \\
+ \text { continentale) }\end{array}$ & inadeguato & inadeguato & inadeguato & inadeguato \\
\hline $\begin{array}{l}\text { Beccamoschino } \\
\text { (mediterranea) }\end{array}$ & favorevole & favorevole & favorevole & favorevole \\
\hline $\begin{array}{l}\text { Usignolo di fiume } \\
\text { (alpina + continentale) }\end{array}$ & favorevole & inadeguato & favorevole & inadeguato \\
\hline $\begin{array}{l}\text { Usignolo di fiume } \\
\text { (mediterranea) }\end{array}$ & favorevole & favorevole & favorevole & favorevole \\
\hline Salciaiola (continentale) & cattivo & cattivo & inadeguato & cattivo \\
\hline Salciaiola (mediterranea) & sconosciuto & cattivo & inadeguato & cattivo \\
\hline Regolo (alpina) & favorevole & favorevole & favorevole & favorevole \\
\hline $\begin{array}{l}\text { Regolo (continentale }+ \\
\text { mediterranea) }\end{array}$ & sconosciuto & sconosciuto & favorevole & sconosciuto \\
\hline $\begin{array}{l}\text { Balia dal collare (alp. e } \\
\text { cont.) }\end{array}$ & cattivo & cattivo & cattivo & cattivo \\
\hline $\begin{array}{l}\text { Balia dal collare } \\
\text { (mediterr.) }\end{array}$ & sconosciuto & cattivo & inadeguato & cattivo \\
\hline $\begin{array}{l}\text { Picchio muraiolo } \\
\text { (alpina) }\end{array}$ & favorevole & sconosciuto & favorevole & favorevole \\
\hline $\begin{array}{l}\text { Picchio muraiolo } \\
\text { (continentale }+ \\
\text { mediterranea) }\end{array}$ & inadeguato & sconosciuto & favorevole & inadeguato \\
\hline $\begin{array}{l}\text { Rampichino alpestre } \\
\text { (alpina) }\end{array}$ & favorevole & favorevole & favorevole & favorevole \\
\hline $\begin{array}{l}\text { Rampichino alpestre } \\
\text { (continentale }+ \\
\text { mediterranea) }\end{array}$ & sconosciuto & sconosciuto & favorevole & sconosciuto \\
\hline Gracchio alpino (alpina) & favorevole & favorevole & favorevole & favorevole \\
\hline $\begin{array}{l}\text { Gracchio alpino } \\
\text { (mediterranea }+ \\
\text { continentale) }\end{array}$ & inadeguato & cattivo & favorevole & cattivo \\
\hline Ciuffolotto (alpina) & favorevole & favorevole & favorevole & favorevole \\
\hline $\begin{array}{l}\text { Ciuffolotto } \\
\text { (mediterranea) }\end{array}$ & favorevole & sconosciuto & favorevole & favorevole \\
\hline Frosone (alpina) & inadeguato & sconosciuto & inadeguato & inadeguato \\
\hline
\end{tabular}




\begin{tabular}{|l|c|c|c|c|}
\hline Specie & Range & Popolazione & Habitat & Complessivo \\
\hline Frosone (mediterranea) & sconosciuto & sconosciuto & sconosciuto & sconosciuto \\
\hline Crociere (alpina) & favorevole & favorevole & favorevole & favorevole \\
\hline $\begin{array}{l}\text { Crociere } \\
\text { (continentale+mediterra } \\
\text { nea) }\end{array}$ & favorevole & inadeguato & favorevole & inadeguato \\
\hline $\begin{array}{l}\text { Zigolo capinero } \\
\text { (continentale) }\end{array}$ & cattivo & cattivo & sconosciuto & cattivo \\
\hline $\begin{array}{l}\text { Zigolo capinero } \\
\text { (mediterranea) }\end{array}$ & sconosciuto & sconosciuto & sconosciuto & sconosciuto \\
\hline $\begin{array}{l}\text { Strillozzo (alpina + } \\
\text { continentale) }\end{array}$ & inadeguato & cattivo & cattivo & cattivo \\
\hline Strillozzo (mediterranea) & favorevole & favorevole & favorevole & favorevole \\
\hline
\end{tabular}

Tab. 6 - Numero di popolazioni per ordine aventi un determinato stato di conservazione complessivo. / Number of populations per order with a given overall conservation status.

\begin{tabular}{|l|r|r|r|r|r|r|r|r|r|}
\hline & Cattivo & \multicolumn{1}{c}{$\boldsymbol{\%}$} & Inadeguato & $\mathbf{\%}$ & Favorevole & $\mathbf{\%}$ & Sconosciuto & \% & Totale \\
\hline Galliformes & 7 & 78 & 2 & 22 & 0 & - & 0 & - & 9 \\
\hline Anseriformes & 3 & 23 & 7 & 54 & 2 & 15 & 1 & 8 & 13 \\
\hline Procellariformes & 2 & 67 & 1 & 33 & 0 & - & 0 & - & 3 \\
\hline Podicipediformes & 0 & - & 0 & - & 2 & 100 & 0 & - & 2 \\
\hline Phoenicopteriformes & 0 & - & 1 & 100 & 0 & - & 0 & - & 1 \\
\hline Ciconiformes & 1 & 50 & 1 & 50 & 0 & - & 0 & - & 2 \\
\hline Pelecaniformes & 4 & 29 & 7 & 50 & 3 & 21 & 0 & - & 14 \\
\hline Accipitriformes & 11 & 50 & 5 & 23 & 6 & 27 & 0 & - & 22 \\
\hline Otidiformes & 1 & 100 & 0 & - & 0 & - & 0 & - & 1 \\
\hline Gruiformes & 3 & 43 & 1 & 14 & 2 & 29 & 1 & 14 & 7 \\
\hline Charadriformes & 7 & 28 & 14 & 56 & 2 & 8 & 2 & 8 & 25 \\
\hline Columbiformes & 0 & - & 2 & 33 & 2 & 33 & 2 & 33 & 6 \\
\hline Cuculiformes & 0 & - & 2 & 100 & 0 & - & 0 & - & 2 \\
\hline Strigiformes & 1 & 10 & 5 & 50 & 4 & 40 & 0 & - & 10 \\
\hline Caprimulgiformes & 0 & - & 1 & 100 & 0 & - & 0 & - & 1 \\
\hline Apodiformes & 0 & - & 1 & 33 & 2 & 67 & 0 & - & 3 \\
\hline Coraciformes & 0 & - & 2 & 50 & 1 & 25 & 1 & 25 & 4 \\
\hline Piciformes & 1 & 12 & 4 & 44 & 4 & 44 & 0 & - & 9 \\
\hline Passeriformes & 35 & 30 & 27 & 23 & 44 & 38 & 10 & 9 & 116 \\
\hline Totale & 76 & 30 & 83 & 33 & 74 & 30 & 17 & 7 & 250 \\
\hline
\end{tabular}




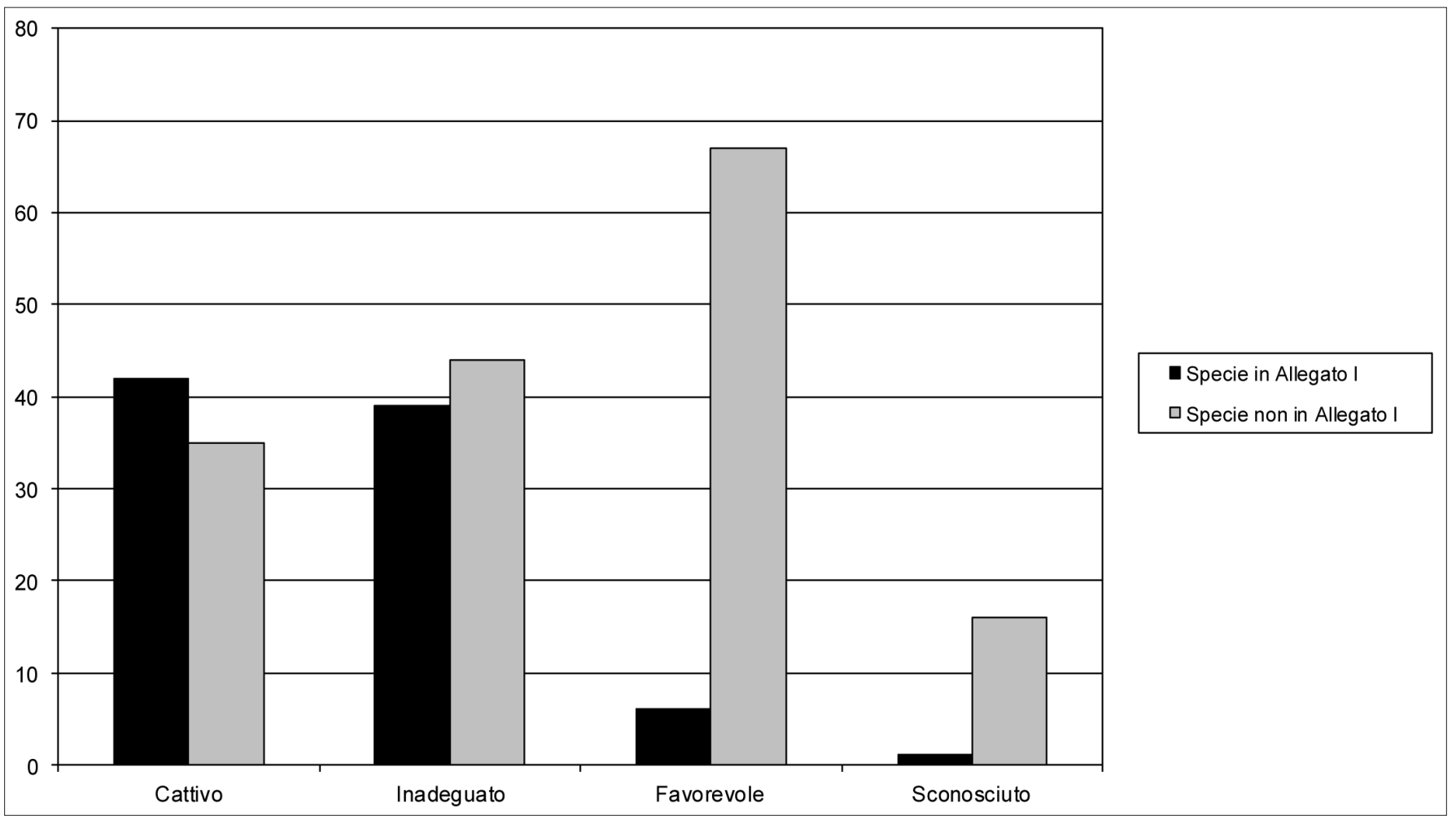

Fig. 1 - Classificazione complessiva delle specie inserite nell'Allegato I Direttiva Uccelli e non, in stato di conservazione cattivo, inadeguato, favorevole e sconosciuto. / Overall classification of species listed in the Annex I of Birds Directive and not, in bad, inadequate, favourable and unknown conservation status.

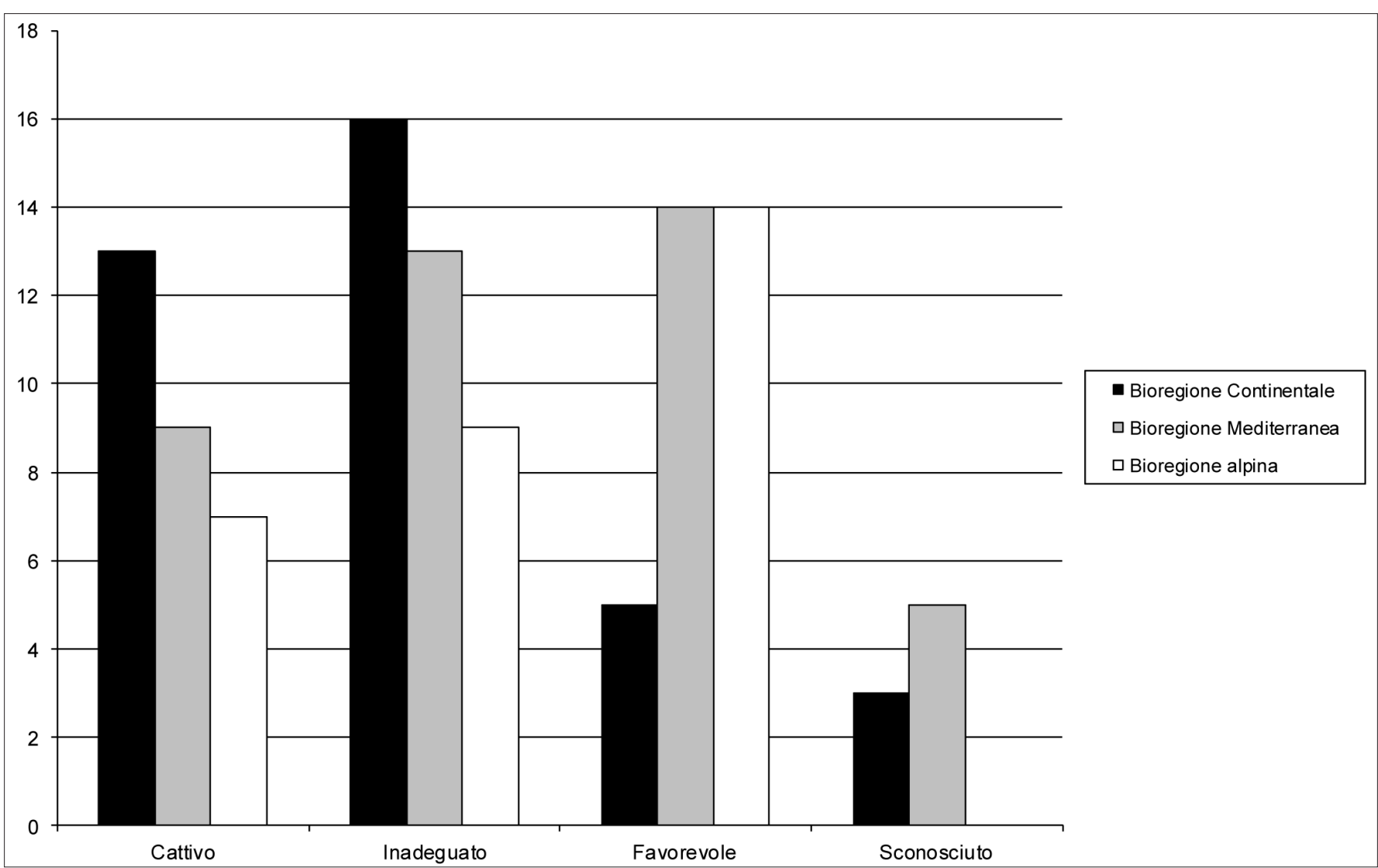

Fig. 2 - Stato di conservazione delle specie ornitiche nelle diverse bioregioni italiane. / Conservation status of avian species in the different Italian bioregions. 


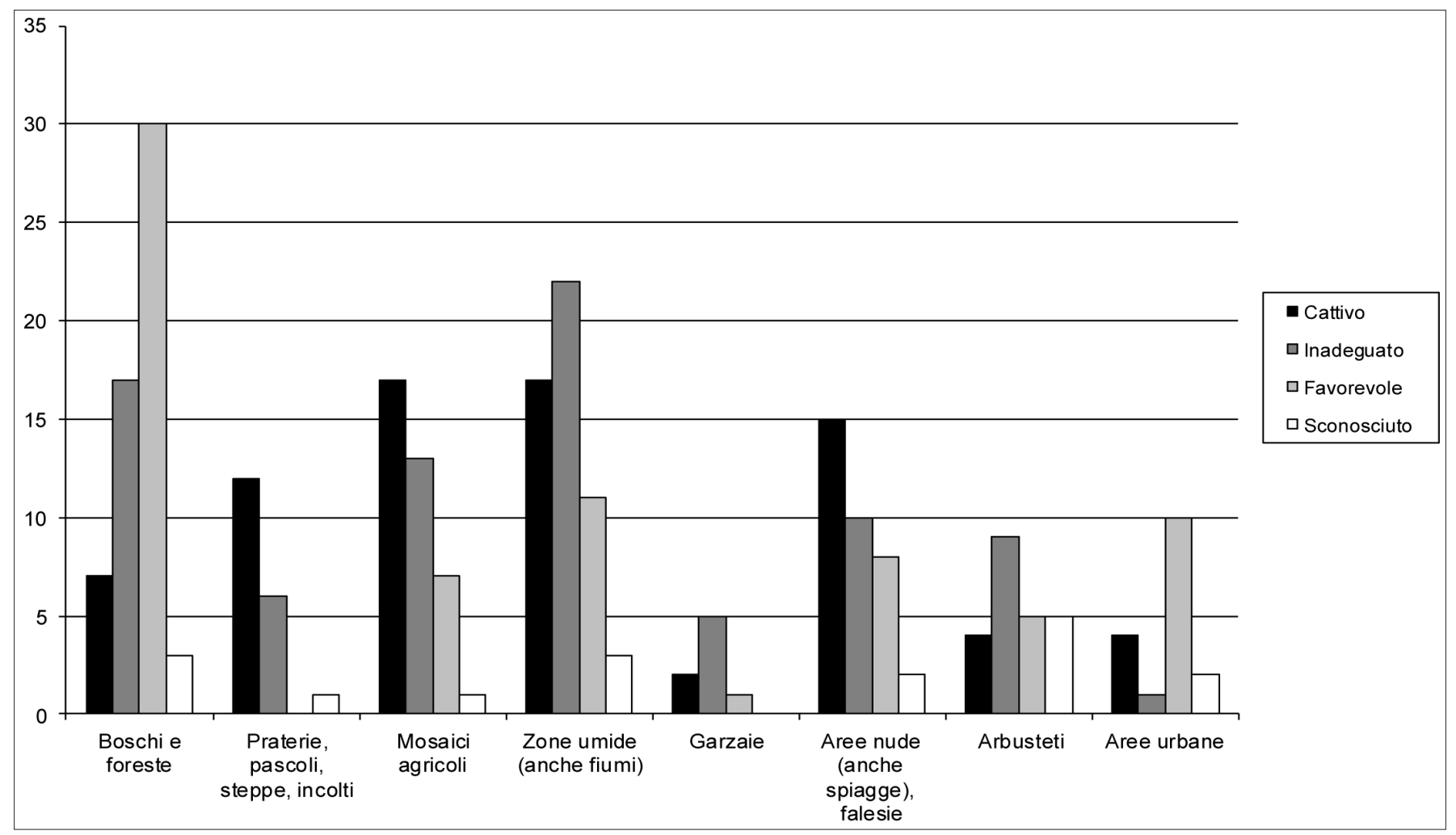

Fig. 3 - Stato di conservazione delle specie nei diversi macro-habitat. / Species conservation status in different macro-habitats.

\section{FRV (FAVOURABLE REFERENCE VALUE)}

Non è stato possibile formulare il FRV per diverse specie per le quali mancano informazioni fondamentali relativamente a parametri demografici e riproduttivi o perché specie di recente colonizzazione o perché specie coloniali e abbondanti: Anseriformes (ad eccezione del Mergus menganser), Alectoris graeca whitakeri, Alectoris rufa, Phasianus colchicus, Calonectris diomedea, Puffinus yelkouan, Phalacrocorax pygmeus, Ciconiformes (ad eccezione della Ciconia ciconia), Phoenicopterus roseus, Podiceps cristatus, Gypaetus barbatus, Gyps fulvus, Circaetus gallicus, Falco naumanni, Falco vespertinus, Rallidae (ad eccezione di Rallus aquaticus, Gallinula chloropus, Fulica atra), Tetrax tetrax, Caradriformes (ad eccezione di Recurvirostra avosetta, Burhinus oedicnemus, Charadius dubius, Charadrius alexandrinus, Vanellus vanellus, Actitis hypoleucos, Tringa totanus, Gelochelidon nilotica, Sterna hirundo), Columba oenas, Clamator glandarius, Strix uralensis, Apodiformes, Merops apiaster, Coracias garrulus, Picus canus, Dryocopus martius. Tra i Passeriformes, Irundinidae, Oenanthe hispanica, Monticola saxatilis, Turdus pilaris, Locustella luscinioides, Acrocephalus shoenobaenus, Acrocephalus melanopogon, Sylvia hortensis, Sylvia sarda, Panurus biarmicus, Tichodroma muraria, Remix pendulinus, Lanius minor, Corvidae (ad eccezione di Nucifraga caryocatactes, Pyrrhocorax pyrrhocorax), Sturnidae, Passer hispaniolensis, Passer montanus, Petronia petronia, Montifringilla nivalis, Serinus serinus, Carduelis citrinella, Carduelis corsicana, Carduelis spinus, Loxia curvirostra, Coccothraustes coccothraustes.
FRV: Specie inserite nell'Allegato I della Direttiva Uccelli

Complessivamente, per 46 popolazioni appartenenti a 20 specie inserite nell'Allegato I della Direttiva Uccelli è stato possibile calcolare un valore di FRV attraverso tecniche di population modelling (Tab. 1).

Per tutte le specie e le popolazioni, il FRV appare superiore alla stima corrente di popolazione, con l'eccezione di alcune popolazioni di Aquila reale Aquila chrysaetos (in aree ove la specie ha raggiunto la capacità portante del territorio, Fasce et al., 2011) e per le principali popolazioni di Falco pellegrino Falco peregrinus, che appaiono al momento in fase di stabilizzazione attorno alla capacità portante dell'ambiente e in recente colonizzazione dell'ambiente urbano da parte di questa specie (Fraissinet, 2008).

Per 15 specie è stato formulato un FRV in termini di densità riproduttiva a una o due scale spaziali.

Per alcune specie, non è stato possibile fornire un FRV in termini di dimensione di popolazione, ma le buone conoscenze sulle densità riproduttive hanno consentito di individuare comunque delle densità favorevoli (es. Martin pescatore Alcedo atthis).

Per 12 specie che hanno recentemente colonizzato (o ri-colonizzato) l'Italia non è stato possibile formulare un FRV.

Per tre specie coloniali, con popolazioni o colonie superiori a 2.500 coppie, non è stato calcolato alcun FRV.

Per 35 specie non è stato possibile calcolare un FRV (tramite population modelling oppure densità riproduttiva), a causa dell'ampio scambio di individui con popo- 
lazioni extra-nazionali e/o in assenza di dati specifici sui tassi di immigrazione ed emigrazione (Gabbiano corso Larus audouinii; Oro et al., 1996), o a causa dell'attuale tasso di mortalità dalla popolazione (Grifone Gyps fulvus in Sardegna; Decandia, 2015), oppure per la mancanza di dati adeguati.

\section{FRV per Specie non in Allegato I Direttiva Uccelli}

Per 10 popolazioni di 6 specie non in Allegato I della Direttiva Uccelli, è stato possibile calcolare un valore di FRV attraverso tecniche di population modelling (Tab. 2).

Per 92 specie è stato formulato un FRV in termini di densità riproduttiva a una o due scale spaziali, con ulteriori suddivisioni per alcune specie in base alla differente idoneità ambientale dei principali contesti di presenza.

\section{STATO DI CONSERVAZIONE}

\section{Specie in Allegato I Direttiva Uccelli}

Su 75 specie di non-Passeriformi, 6 hanno uno stato di conservazione favorevole ( $8 \%$ ), 37 uno stato di conservazione inadeguato (49\%), mentre $32(43 \%)$ mostrano uno stato di conservazione cattivo (Tab. 3).

$\mathrm{Su} 13$ specie di Passeriformi considerate, 10 (78\%) hanno uno stato di conservazione cattivo, 2 (15\%) inadeguato, $1(7 \%)$ sconosciuto, nessuna specie risulta con uno status favorevole di conservazione.

\section{Specie non incluse nell'Allegato I Direttiva Uccelli}

Su 59 specie di non-Passeriformi, 23 (39\%) hanno uno stato di conservazione favorevole, 19 (32\%) uno stato di conservazione inadeguato, $10(17 \%)$ mostrano uno stato di conservazione cattivo e 7 (12\%) sconosciuto.

Su 102 specie di Passeriformi considerate, 46 (45\%) hanno uno stato di conservazione 'favorevole', 28 (27\%) uno stato di conservazione 'inadeguato', 18 (18\%) mostrano uno stato di conservazione 'cattivo' e 10 'sconosciuto'.

Il $55 \%$ delle specie in cattivo stato di conservazione è caratteristica delle zone agricole (Fig 3).

Significativo, tra le specie di Passeriformi in cattivo stato di conservazione, il numero relativamente elevato di specie legate alle zone umide (Basettino Panurus biarmicus, Pendolino Remiz pendulinus, Migliarino di palude Emberiza shoeniclus, Salciaiola Locustella luscinioides, Forapaglie comune Acrocephalus shoenobaenus), così come evidenziato in Fig. 3.

Le lacune conoscitive relative ai Passeriformi sono evidenziate dalle 11 specie con stato di conservazione generale sconosciuto: per queste specie, per due voci delle tre considerate (Passera europea Passer domesticus, Passera sarda Passer hispaniolensis, Venturone alpino Carduelis citrinella, Venturone corso Carduelis corsicana, Lucherino Carduelis spinus, Zigolo muciatto Emberiza cia) oppure per tutte e tre le voci (Sterpazzola della Sardegna Sylvia conspicillata, Zigolo capinero Emberiza melanocephala) non è stato possibile valutare lo stato di conservazione a causa della scarsità di informazioni relative a questi taxa.

\section{Considerazioni sulle popolazioni nelle Regioni biogeografiche}

Così come evidenziato in Fig. 2, la bioregione continentale ospita il maggior numero di specie in cattivo stato di conservazione, molte delle quali legate alle zone agricole (Barbagianni Tyto alba, Torcicollo Jynx torquilla, Rondine Hirundo rustica, Cappellaccia Galerida cristata, Zigolo capinero, Strillozzo Emberiza calandra), o alle zone umide (Tarabuso Botaurus stellaris, Salciaiola), mentre la bioregione alpina e mediterranea ospitano il maggior numero di specie con stato di conservazione favorevole (Fig. 2).

Al contrario, la maggior parte delle specie con stato di conservazione sconosciuto sono localizzate nella bioregione mediterranea (Merlo dal collare Turdus torquatus, Regolo Regulus regulus, Frosone Coccothraustes coccothraustes, Rampichino alpestre Certhia familiaris, Zigolo capinero), verosimilmente la bioregione che presenta ancora le principali lacune conoscitive.

\section{DISCUSSIONE}

La Direttiva Uccelli ha avuto un impatto positivo nelle politiche di conservazione del nostro Paese, così come evidenziato anche a livello internazionale (Donald et al., 2007; Sanderson et al., 2016), introducendo vincoli e misure più efficaci per la tutela e la gestione delle specie, anche attraverso la designazione delle ZPS e incentivando al tempo stesso il miglioramento della conoscenza sulle popolazioni di uccelli (in particolare delle specie in All. I) ed il loro monitoraggio. Tuttavia, molte delle specie in Allegato I alla Direttiva Uccelli, soffrono ancora di un cattivo stato di conservazione (ad es. Capovaccaio Neophron percnopterus, Re di quaglie Crex crex, Gallo cedrone Tetrao urogallus, Pernice bianca Lagopus muta, Averla piccola Lanius collurio, Calandra Melanocorypha calandra, Calandrella Calandrella brachydactyla).

Le sette specie con stato di conservazione favorevole, ovvero Garzetta Egretta garzetta, Airone rosso Ardea purpurea, Falco pecchiaiolo Pernis apivorus, Falco di palude Circus aeruginosus, Falco pellegrino, Cavaliere d'Italia Himantopus himantopus e Picchio cenerino Picus canus, hanno mostrato tutte ampliamenti recenti più o meno evidenti del range distributivo, incremento demografico o prevalente stabilità e occupano ambienti ritenuti nel complesso stabili oppure in locale incremento (Nardelli et al., 2015).

Critico appare il quadro relativo ai Galliformi, con 5 specie su 7 con stato di conservazione cattivo. Oltre alla sospensione dell'attività venatoria (Coturnice Alectoris graeca saxatilis, Pernice bianca Lagopus muta helvetica, Fagiano di monte Lyrurus tetrax), sarebbero auspicabili per tali specie singole misure di conservazione per contrastarne il declino, stanti anche le problematiche prossime future legate alle modifiche ambientali provocate dai cambiamenti climatici (Imperio et al., 2013). 
Preoccupante lo stato della Berta minore Puffinus yelkouan e della Berta maggiore Calonectris diomedea, non solo per le condizioni generalmente avverse, relative soprattutto all'habitat, e al basso successo riproduttivo dovuto alle specie aliene (cfr. Ratto nero Rattus rattus, Capizzi et al., 2010). La responsabilità nazionale per la conservazione di queste specie è altissima: la prima risulta endemica del Mediterraneo e presente con circa il $67,3 \%$ della popolazione globale in Italia (e risulta in calo anche negli altri paesi mediterranei inclusi nell'areale distributivo della specie; Bourgeois \& Vidal, 2008), mentre la popolazione italiana di Berta maggiore, rappresenta il 4,7\% di quella europea) (http://bd.eionet.europa.eu/article12/progress?period=1\&conclusion=bs).

Così come confermato anche dalla Lista Rossa Nazionale relativamente al rischio di estinzione (Peronace et al., 2012), tutte le specie di avvoltoi presenti in Italia si trovano in un cattivo stato di conservazione, a causa della ridotta popolazione e del rischio di saturnismo (Gipeto; Bassi et al., 2014), di perdita di habitat, persecuzione diretta, elettrocuzione, impatto con le torri eoliche, trasformazione dei pascoli e, non ultimo, uso di bocconi avvelenati (Grifone), minaccia quest'ultima che riguarda anche altre specie necrofaghe in cattivo stato di conservazione, come il Nibbio reale Milvus milvus (Allavena et al., 2006).

Nessun Passeriforme inserito in Allegato I ha uno stato di conservazione favorevole a scala nazionale. Questo risultato evidenzia come queste specie, spesso oggetto di minor attenzione anche in ambito conservazionistico (cfr. Brambilla et al., 2013), versino in una condizione estremamente preoccupante (Nardelli et al., 2015).

Alcune di esse hanno in Italia una frazione assolutamente preponderante della loro popolazione europea o addirittura globale: ad esempio, quasi un terzo della popolazione complessiva di Magnanina sarda Sylvia sarda, una specie il cui stato di conservazione è sconosciuto, nidifica in Italia.

Alcune specie di Alaudidi (Calandra Melanocorypha calandra, Calandrella Calandrella brachydactyla) mostrano un cattivo stato di conservazione conseguente ai cambiamenti di uso del suolo avvenuti soprattutto nelle ultime decadi, ed in particolare all'abbandono delle pratiche agro-pastorali tradizionali (Massa \& La Mantia, 2010; La Mantia et al., 2014).

Entrambe le specie di averla (Averla piccola Lanius collurio e Averla cenerina Lanius minor) si trovano in cattivo stato di conservazione, soprattutto a causa dell'intensificazione dell'agricoltura e dell'abbandono dei paesaggi agricoli tradizionali non intensivi, cambiamenti che portano alla scomparsa del mosaico idoneo a queste specie e comprendente piccole aree cespugliate, porzioni incolte, siepi e prati semi-naturali (Brambilla et al., 2007b; 2007c; Casale \& Brambilla, 2009; Brambilla et al., 2009, 2010; Ceresa et al., 2012; Chiatante et al., 2014).

Per le specie non inserite nell'Allegato I della Direttiva Uccelli, la situazione è sostanzialmente migliore. Questa differenza è legata probabilmente all'inserimento nell' Allegato I della Direttiva Uccelli di specie più minacciate e pertanto, aventi generalmente stato di conservazione non favorevole anche a livello di singoli stati membri (Donald et al., 2007). Il 50\% delle 10 specie in cattivo stato di con- servazione sono specie cacciabili secondo la normativa nazionale; per alcune di esse (ad esempio Starna Perdix perdix, Pernice rossa Alectoris rufa, Quaglia Coturnix coturnix) l'attività venatoria riveste indubbiamente un ruolo impattante, sia attraverso meccanismi diretti (abbattimento e disturbo) che indiretti (rilasci di individui appartenenti ad altre sottospecie o specie simili con conseguente ibridazione ed inquinamento genetico e modifica delle abitudini ecologiche), confermando il quadro particolarmente critico dei Galliformi, così come evidenziato anche per le specie in Allegato I della Direttiva Uccelli. Anche due specie di Anseriformi, quali Marzaiola Anas querquedula e Moriglione Aythya ferina, risultano in cattivo stato di conservazione; il Moriglione è inoltre inserito nella categoria vulnerable della Lista Rossa Europea (Birdife International, 2015).

Per alcune specie, quali Canapiglia Anas strepera, Porciglione Rallus aquaticus, Gabbiano comune Chroicocephalus ridibundus, Colombella Columba oenas, Upupa Upupa epops vi sono uno o più criteri tra i tre considerati (habitat, range popolazione) classificati come "sconosciuto". In questi casi, un miglioramento di conoscenze, quale, ad esempio, il semplice monitoraggio annuale delle colonie riproduttive nel caso del Gabbiano comune, potrebbe determinare un cambiamento nella valutazione generale dello stato di conservazione ed il giudizio espresso relativamente a queste specie deve pertanto essere considerato come provvisorio. Nel caso della Beccaccia Scolopax rusticola, invece, la difficoltà di contattare la specie e quindi di monitorarne trend, variazioni areali e cambiamenti nella qualità e quantità dell'habitat, in un contesto caratterizzato da presenza irregolare e sparsa, è alla base dell'attribuzione del giudizio "sconosciuto" a tutte le tre voci considerate per la valutazione dello stato di conservazione.

Ancor più lacunosa è la conoscenza per diverse specie di Passeriformi: nel caso di 11 specie (Cappellaccia $\mathrm{Ga}$ lerida cristata, Sordone Prunella collaris, Sterpazzolina comune Sylvia cantillans, Sterpazzolina di Moltoni Sylvia subalpina, Bigiarella Sylvia curruca, Beccafico Sylvia borin, Pigliamosche Muscicapa striata, Picchio muraiolo Tichodroma muraria, Ciuffolotto Pyrrhula pyrrhula), per le quali è stato possibile determinare lo stato di conservazione complessivo, vi è comunque una voce tra le tre considerate classificata come stato "sconosciuto". Anche in questi casi, un miglioramento delle conoscenze potrebbe determinare un cambiamento nella valutazione generale dello stato di conservazione e il giudizio espresso relativamente a queste specie deve pertanto essere preso come provvisorio. Particolare è il caso di diverse specie che erano un tempo relativamente comuni ed oggi appaiono in precario stato di conservazione (ad es. Averla capirossa Lanius senator, Allodola Alauda arvensis, Saltimpalo Saxicola torquatus, Bigia grossa, Passera d'Italia Passer italiae), evidenziando la necessità di un'implementazione più forte della Direttiva Uccelli.

Per conservare le specie ornitiche, infatti, quest'ultima ha individuato nuovi ambiti verso i quali privilegiare l'erogazione di finanziamenti per progetti (ad es. progetti LIFE, Misure agro-ambientali dei Piani di Sviluppo Rurale), indirizzati alla realizzazione di azioni pratiche e 
strettamente mirate a favorire le specie ornitiche, sia le specie comuni che quelle in Allegato I, e contribuendo al graduale e crescente sviluppo di una "cultura della conservazione", oggi maggiormente radicata nella società. Molte specie, sia in Allegato I che non, rimangono in uno stato di conservazione non favorevole $(28,1 \%$ del totale risulta in stato cattivo), e molte specie di Passeriformi $(27,4 \%$ del totale risulta in stato cattivo) sono andate incontro a pesanti cali di popolazione, come ha evidenziato anche la recente Lista Rossa nazionale (Peronace et al., 2012). D'altro canto, è innegabile che molte popolazioni considerate maggiormente in pericolo per la loro limitata consistenza numerica e ridotta distribuzione (specie di Laridae e Sternidae ad es.) hanno potuto usufruire negli ultimi decenni di un più elevato livello di protezione, in particolare per quanto riguarda le specie acquatiche nidificanti e svernanti, in cui ha effettivamente pesato maggiormente, rispetto ad altri contesti ambientali, la maggiore tutela e la superficie nel complesso più ridotta degli habitat occupati (Nardelli et al., 2015). Al contrario, le ampie superfici agricole di pianura si presentano come i contesti ambientali più poveri di specie e in cui si annoverano molte di quelle con cattivo stato di conservazione (Rete Rurale Nazionale \& LIPU, 2015).

È evidente che il destino di molte specie ornitiche, tra cui molte di interesse conservazionistico e a maggiore vulnerabilità o rischio di estinzione, è vincolato a sistemi sottoposti a una più diretta influenza antropica. Pertanto, le strategie per mantenere popolazioni in grado di autosostenersi o per migliorarne l'andamento demografico, dovranno sempre più interfacciarsi con la gestione di questi contesti ambientali.

L'art. 2 della Direttiva Uccelli obbliga gli Stati membri a mantenere lo stato di conservazione favorevole a scala nazionale.

Il valore di riferimento favorevole è, in questo senso, il parametro quantitativo chiave per definire la soglia per lo stato di conservazione favorevole di una determinata specie. Questa valutazione è stata introdotta nel processo di Reporting dell'art. 17 della Direttiva Habitat e si articola in FRV per la popolazione, l'habitat della specie e il suo range. Anche le prospettive per la specie vengono incluse nella valutazione. In questo lavoro, i modelli di PVA sono stati utilizzati per identificare popolazioni "ideali" secondo i differenti contesti, ad esempio popolazioni minime vitali per specie/popolazioni ad elevato rischio di estinzione, oppure popolazioni sufficientemente ampie da assicurare persistenza a lungo termine anche in condizioni molto sfavorevoli nel caso di popolazioni attualmente non a rischio di estinzione. Ad oggi, l'Italia risulta essere uno dei pochi paesi europei (insieme a Belgio e Cipro) (Tye et al., 2014), che ha formulato una valutazione dello stato di conservazione complessiva delle specie nidificanti all'interno del proprio territorio (Brambilla et al., 2011), in cui sono state considerate sia le specie in Allegato I della Direttiva Uccelli (2009/147/CE) (Gustin et al., 2009), che le altre nidificanti (Gustin et al., 2010a; 2010b). Recentemente è stato predisposto un metodo per la definizione del valore di riferimento per l'habitat (FRV-H o HRV), contraddistinto da due componenti differenti, l'estensione e la qualità (Brambilla et al., 2012; 2014).
In questo lavoro, per una significativa frazione delle specie nidificanti in Italia è stato fornito un FRV in termini di popolazione (o di meta-popolazione o di livello biogeografico - alpino, continentale e mediterraneo) oppure in termini di densità riproduttiva, rappresentando un progetto 'pilota' anche in termini metodologici, non solo a scala nazionale, ma anche europea.

La definizione del FRV basata su valori di densità riproduttiva ha un valore 'parziale': esso può essere utilizzato agevolmente come termine di riferimento per singoli siti o gruppi di siti, ma non fornisce un termine di confronto direttamente applicabile all'intera scala nazionale. Se gli auspicati sforzi per quantificare range attuale, storico e/o potenziale di ciascuna specie, in base ad una quantificazione dell'habitat potenzialmente idoneo venissero attuati, sarebbe possibile tradurre questo valore di densità in stime di popolazione. Tuttavia, l'indicazione di valori di densità ideali a diverse scale spaziali rappresenta di per sé un utile strumento di gestione, a livello di sito Natura 2000 e di comprensorio.

Sebbene concepito e sviluppato a scala nazionale, il presente lavoro può rappresentare quindi uno strumento utile per pianificare la conservazione delle specie ornitiche anche a scala regionale. Il metodo applicato, infatti, è potenzialmente ri-applicabile anche a realtà regionali, soprattutto nel caso di specie con distribuzione localizzata o esclusiva in una o poche regioni. Una prima sperimentazione in questo senso è avvenuta in Lombardia nell'ambito del Progetto LIFE+ GESTIRE (LIFE11 NAT/ IT/000044), al cui interno è stato condotto un approfondimento dei metodi per calcolare i valori di riferimento a scala regionale ed è stato valutato lo stato di conservazione a livello regionale (http://www.naturachevale.it/it/).

Alcune delle specie per le quali le informazioni concernenti la dinamica e consistenza delle popolazioni sono particolarmente scarse, hanno in Italia una frazione assolutamente preponderante della loro popolazione europea o addirittura globale: è il caso della Sterpazzolina di Moltoni Sylvia subalpina (l'Italia ospita circa i due terzi della popolazione globale della specie) o del Venturone corso Carduelis corsicana (circa il 17\% della popolazione globale della specie nidifica in Italia). La conservazione di queste specie nel nostro paese assume quindi particolare importanza a livello mondiale ed è urgente colmare le lacune conoscitive ancora presenti.

Per molte specie per le quali un FRV potrebbe essere ottenuto con un'adeguata conoscenza dei parametri riproduttivi, la mancanza delle informazioni necessarie ne impedisce la formulazione. Tali lacune condizionano un gran numero di famiglie (soprattutto quelle poco studiate come ad esempio Rallidae e Picidae) e alcune specie target che rappresentano una priorità di conservazione. In ogni caso, la densità riproduttiva è ampiamente adottata come una misura indiretta della disponibilità di habitat e potrebbe essere presa come primo step nella misura dello stato di conservazione, indirizzando il monitoraggio della specie a larga scala.

Si evidenzia come molte delle numerose specie in uno stato di conservazione favorevole, per rimanere tali, richiedano un'attenta strategia di conservazione e/o di gestione a livello nazionale, come recentemente evidenziato nel Reporting sulla direttiva Uccelli (Nardelli et al., 2015). 
Infine, occorre rimarcare come il fatto che diverse specie siano attualmente al riparo dal rischio di estinzione a breve termine, non comporta che esse si trovino "automaticamente" in buono stato di conservazione, condizione per la quale è necessario che la specie abbia un ruolo "significativo" nel proprio habitat di riferimento, rinvenendosi con frequenze e densità soddisfacenti e ricoprendo le funzioni ecologiche che le sono proprie.

Le forti pressioni cui molte specie e popolazioni sono sottoposte, quali i cambiamenti climatici, il continuo degrado ambientale che caratterizza molti ecosistemi, le variazioni ad ampia scala nella dinamica di popolazione di molte specie, rende ancora più complessa la valutazione dello stato di conservazione delle specie. Non si può quindi ritenere una specie o una popolazione in buono stato di conservazione solamente alla luce di un valore di popolazione/densità pari o superiore al $\mathrm{FRV}$, ma occorre valutare accuratamente le minacce e pressioni cui la specie/popolazione è soggetta o potrà esserlo nel prossimo futuro (con particolare riferimento alle warning lights sopra descritte).

Risulta infine evidente come i FRV dovranno essere sottoposti a periodica rivalutazione ed aggiornamento, sulla base soprattutto dei nuovi dati che ogni sei anni vengono forniti dal Reporting sull'applicazione della Direttiva Uccelli 147/2009CE (Nardelli et al., 2015).

In alcuni casi, il FRV può (e in un certo senso deve) essere formulato considerando popolazioni che occupano aree che vanno al di là dei confini amministrativi (in questo caso nazionali). A titolo di esempio, il target di popolazione proposto per il Gipeto (per il quale non viene calcolato un FRV, essendo la popolazione di recente reinsediamento) è stato formulato a scala alpina (Gustin et al., 2009). Appare chiara quindi, la necessità di coordinare e raccordare gli sforzi con paesi confinanti nel caso di popolazioni 'condivise' da più stati.

Nel presente lavoro non sono stati sviluppati FRV per le specie non nidificanti. Cionondimeno, il principio dell'individuazione di valori di riferimento può essere applicato anche a popolazioni esclusivamente migratrici e/o svernanti. La formulazione del FRV per queste specie/popolazioni dovrà basarsi sullo sviluppo di un metodo dedicato, che tenga conto delle variazioni nella distribuzione, presenza ed abbondanza di tali specie non direttamente legate al loro stato di conservazione (o a quello dei loro habitat), ma a fattori quali le condizioni climatiche e l'andamento stagionale.

\section{BIBLIOGRAFIA}

Alessandria G., Carpegna F. \& Della Toffola M., 2001 Il Cormorano Phalacrocorax carbo nella regione piemontese. Parte II. Distribuzione e biologia della popolazione nidificante (Aves, Phalacrocoracidae). Rivista Piemontese di Storia Naturale, 22: 261-280.

Allavena S., Andreotti A., Angelini J. \& Scotti M. 2006. Status e conservazione del Nibbio reale e Nibbio bruno in Italia e in Europa meridionale. In: Status e conservazione del Nibbio reale e Nibbio bruno in Italia e in Europa meridionale, S. Maria del Mercato, Serra S. Quirico 11-12 marzo 2006: 4-5.
Allavena S., Panella M., Pellegrini M. \& Zocchi A., 1991 L'Aquila reale nell'Appennino centrale: distribuzione, andamento riproduttivo e problemi di conservazione. Supplemento alle Ricerche di Biologia della Selvaggina, XVI: 259-262.

Aluigi A., Fasano S. \& Toffoli R., 2014 - Densità riproduttiva del Succiacapre Caprimulgus europaeus in aree della rete Natura 2000 in Sicilia. In: Atti XVI Convegno Italiano di Ornitologia. Cervia (RA), 2225 settembre 2011. Tinarelli R., Andreotti A., Baccetti N., Melega L., Roscelli F., Serra L., \& Zenatello M., (eds). Scritti, studi e Ricerche di Storia Naturale della Repubblica di San Marino: 129-130.

Amici A., Serrani F., Adriani S., Ronchi B., Bonanni M. \& Primi R., 2012 - Uso del modello di Idoneità di sito per la nidificazione (MISN) per la stima dei parametri di popolazione della Coturnice appenninica, Alectoris graeca orlandoi, nelle province di Rieti e Frosinone, aggiornata al dicembre 2009. Rivista italiana di Ornitologia, 82: 75-79.

Andreotti A. \& Leonardi G. (a cura di), 2009 - Piano d'azione nazionale per il Capovaccaio Neophron percnopterus. Ministero dell'Ambiente. ISPRA. Quaderni di Conservazione della Natura, 30.

Andreotti A., Leonardi G., Sarà M., Brunelli M., De Lisio L., De Sanctis A., Magrini M., Nardi R., Perna P. \& Sigismondi A., 2008 - Landscape-scale spatial distribution of the Lanner Falcon (Falco biarmicus feldeggii) breeding population in Italy. Ambio, 37: 440-444.

Angelini J., 2014 - Status del Gracchio corallino Pyrrhocorax pyrrhocorax e del Gracchio alpino Pyrrhocorax graculus nella regione Marche, Italia centrale. In: Atti XVI Convegno Italiano di Ornitologia. Cervia (RA), 22-25 settembre 2011. Tinarelli R., Andreotti A., Baccetti N., Melega L., Roscelli F., Serra L.\& Zenatello M., (eds). Scritti, studi e Ricerche di Storia Naturale della Repubblica di San Marino: 281-282.

Arroyo B. \& Ferreiro E., 1997 - European Union Species Action Plan for Bonelli's Eagle (Hieraaetus fasciatus). European Commission, Brussels.

Artuso I., 2003 - Consistenza e densità del Fagiano di monte Tetrao tetrix e della Coturnice Alectoris grae$c a$ in Val Brembana (BG) (1996-2002). Avocetta, 27: 97.

Bassi E., Ferloni M., Gugiatti A., Pedrotti L., Di Giancamillo M. \& Grilli G., 2014 - Il rischio di saturnismo negli uccelli necrofagi in relazione alle attuali modalità di caccia degli ungulati. In: Atti XVI Convegno Italiano di Ornitologia. Cervia (RA), 22-25 settembre 2011. Tinarelli R., Andreotti A., Baccetti N., Melega L., Roscelli F., Serra L. \& Zenatello M., (eds). Scritti, studi e Ricerche di Storia Naturale della Repubblica di San Marino: 450-457.

Bionda R. \& Bordignon L., 2006 - Atlante degli uccelli nidificanti del Verbano Cusio Ossola. Quaderni di natura e paesaggio del VCO, 6. Provincia del Verbano Cusio Ossola.

BirdLife International, 2004 - Birds in Europe: population estimates, trends and conservation status. BirdLife 
International. BirdLife Conservation Series, Cambridge, 12.

BirdLife International, 2015 - European red list of birds. Office for Official Publications of the European Communities, Luxembourg.

Boano G., Brichetti P., Cambi D., Meschini E., Mingozzi T. \& Pazzucconi A., 1985 - Contributo alla conoscenza dell'avifauna della Basilicata. Ricerche di Biologia della Selvaggina, 75: 1-37.

Boano A., Brunelli M., Bulgarini F., Montemaggiori A., Sarrocco S. \& Visentin M., 1995 - Atlante degli uccelli nidificanti nel Lazio. Alula, 2 (1-2): 29-30.

Bocca M., 1985 - Il Fagiano di monte Tetrao tetrix in Valle d'Aosta, dati preliminari sugli studi in corso. In: Fasola M. (ed.). Atti III Convegno italiano di Ornitologia: 42-44.

Bogliani G., Sergio F. \& Tavecchia G., 1999 - Woodpigeons nesting in association with hobby falcons: advantages and choice rules. Animal behaviour, 57: 125131.

Bon M., Scarton F., Stival E., Sattin L., \& Sgorlon G. (eds.), 2014 - Nuovo Atlante degli Uccelli nidificanti e svernanti in provincia di Venezia. Associazione Faunisti Veneti, Museo di storia Naturale di Venezia.

Bordignon L., 2004 - Gli uccelli della provincia di Novara. Provincia di Novara.

Borgo A., 2013 - Densità di Astore Accipiter gentilis, Falco pellegrino Falco peregrinus, Civetta nana Glaucidium passerinum e Civetta capogrosso Aegolius funereus nel Friuli occidentale. In: Atti Secondo Convegno Italiano Rapaci diurni e Notturni, Treviso, 12-13 ottobre 2012. Mezzavilla F., Scarton F., (a cura di). Associazione Faunisti Veneti, Quaderni Faunistici, 3: 282-286.

Borlenghi F. \& Corsetti L., 2001 - Densità e fattori limitanti dell'Aquila reale nell'Appennino centrale. Rivista italiana di Ornitologia, 72: 19-26.

Borlenghi F. \& Corsetti L., 2004 - L'Aquila reale, Aquila chrysaetos, nel Lazio. In: Uccelli rapaci nel Lazio: status e distribuzione, strategie di conservazione. Corsetti L., (ed). Atti del Convegno, Sperlonga 13 dicembre 2003. Ed. Belvedere, Latina: 33-38.

Bourgeois K. \& Vidal E., 2008 - The endemic Mediterranean yelkouan shearwater Puffinus yelkouan: distribution, threats and a plea for more data. Oryx, 42 (2): 187-194.

Boyce M.S., 1992 - Population viability analysis. Annual review of ecology and Sistematics, 23: 481-506.

Brambilla M., 2003 - Densità riproduttiva e invernale del Porciglione Rallus aquaticus in una zona umida della Lombardia. Avocetta, 27: 151.

Brambilla M. \& Rubolini D., 2004 - Water Rail Rallus aquaticus breeding density and habitat preferences in northern Italy. Ardea, 92: 11-17.

Brambilla M. \& Jenkins R.K.B., 2009 - Cost-effective estimates of Water Rail Rallus aquaticus breeding population size. Ardeola, 56: 95-102.

Brambilla M. \& Rubolini D., 2009 - Intra-seasonal changes in distribution and habitat associations of a multi-brooded bird species: implications for conservation planning. Animal Conservation, 12: 71-77.
Brambilla M., Casale F. \& Siccardi P., 2007 - Linee guida e indicazioni gestionali per laconservazione dell'Averla piccola (Lanius collurio), specie di interesse comunitario (Allegato I, Direttiva 79/409/CEE), in Lombardia. Fondazione Lombardia per l'Ambiente, Milano.

Brambilla M., Casale F., Bergero V., Bogliani G., Crovetto G.M, Falco R., Roati M. \& Negri I., 2010 - Glorious past, uncertain present, bad future? Assessing effects of land-use changes on habitat suitability for a threatened farmland bird species. Biological Conservation, 143: 2770-2778.

Brambilla M., Casale F., Bergero V., Crovetto G.M., Falco R., Negri I., Siccardi P. \& Bogliani G., 2009 GIS-models work well, but are not enough: Habitat preferences of Lanius collurio at multiple levels and conservation implications. Biological Conservation, 142: 2033-2042.

Brambilla M., Celada C. \& Gustin M., 2014 - Setting Favourable Habitat Reference Values for breeding birds: general principles and examples for passerine birds. Bird Conservation International, 21: 107-118.

Brambilla M., Gustin M. \& Celada C., 2011 - Defining favourable reference values for bird populations in Italy: setting long-term conservation targets for priority species. Bird International Conservation, 21:107118.

Brambilla M., Quaglierini A., Reginato F., Vitulano S. \& Guidali F., 2008 - Syntopic taxa in the Sylvia cantillans species complex. Acta Ornithologica, 43: 217220.

Brambilla M., Reginato F. \& Guidali F., 2007 - Habitat use by Moltoni's Warbler Sylvia cantillans moltonii in Italy. Ornis Fennica, 84: 91-96.

Brambilla M., Rubolini D. \& Guidali F., 2007 - Between land abandonment and agricultural intensification: habitat preferences of Red-backed Shrikes Lanius collurio in low-intensity farming conditions. Bird Study, 54: 160-167.

Brambilla M., Tellini Florenzano G., Sorace A. \& Guidali F., 2006 - Geographical distribution of Subalpine Warbler Sylvia cantillans subspecies in mainland Italy. Ibis, 148: 568-571.

Brichetti P. \& Fasola M. (ed.), 1990 - Atlante degli uccelli nidificanti in Lombardia (1983-1987). Editoriale Ramperto. Brescia.

Brichetti P., De Franceschi P. \& Baccetti N., 1992 - Fauna d'Italia. Aves. I. Gaviidae-Phasianidae. Edizioni Calderini, Bologna.

Brichetti P. \& Fracasso G., 2003 - Ornitologia Italiana Vol. 1. Gaviidae-Falconidae. Alberto Perdisa Editore, Bologna.

Brichetti P. \& Fracasso G., 2004 - Ornitologia Italiana Vol. 2. Tetraonidae-Scolopacidae. Alberto Perdisa Editore, Bologna.

Brichetti P. \& Fracasso G., 2006 - Ornitologia Italiana Volume 3. Stercorariidae-Caprimulgidae. Alberto Perdisa Editore, Bologna.

Brichetti P. \& Fracasso G., 2007 - Ornitologia Italiana. Vol. 4. Apodidae-Prunellidae. Alberto Perdisa Editore, Bologna. 
Brichetti P. \& Fracasso G., 2008 - Ornitologia Italiana. Volume 5. Turdidae-Cisticolidae. Alberto Perdisa Editore, Bologna.

Brichetti P. \& Grattini N., 2008 - Distribuzione, consistenza ed evoluzione delle popolazioni di Basettino Panurus biarmicus nidificanti in Italia nel periodo 1980-2006. Avocetta, 32: 47-53.

Brichetti P. \& Fracasso G., 2010 - Ornitologia Italiana Vol. 6. Sylviidae- Paradoxornithidae. Oasi Alberto Perdisa Editore, Bologna.

Brichetti P. \& Grattini N., 2010 - Distribuzione e trend delle popolazioni di pendolino Remiz pendulinus nidificanti in Italia nel periodo 1980-2007. Picus, 36 (69): $1-11$.

Brichetti P. \& Grattini N., 2013 - Distribuzione, consistenza ed evoluzione delle popolazioni di Migliarino di palude, Emberiza shoeniclus, nidificanti in Italia nel periodo 1980-2010. Rivista italiana di Ornitologia, 81 (2): 97-109.

Brichetti P. \& Fracasso G., 2014 - Check-list degli Uccelli italiani aggiornata al 2014. Rivista italiana di Ornitologia, 85 (1): 31-50.

Brugnoli A. \& Masé R., 1998 - Gestione venatoria della coturnice in provincia di Trento. Centro di Ecologia Alpina report, 15: 1-120.

Brunelli M., Sarrocco S., Corbi F., Sorace A., Boano A., De Felici S., Guerrieri G., Meschini A. \& Roma S. (a cura di), 2011 - Nuovo atlante degli uccelli nidificanti nel Lazio. Edizioni ARP (Agenzia regionale Parchi), Roma.

Bulgarini F. \& Visentin M., 1995 - Successo riproduttivo dell'Albanella minore nelle colture cerealicole del Lazio. Avocetta, 19: 112.

Cairo E., 2008 - Indagine sulle popolazioni nidificanti di Codirosso Phoenicurus phoenicurus e di Codirosso spazzacamino Phoenicurus ochruros nella Città di Bergamo. Ecologia Urbana, 20: 21-25.

Caldonazzi M., Marsilli A., Torboli C. \& Zanghellini S., 2001 - L'utilizzo dei nidi artificiali per Passeriformi in coltivazioni di melo della provincia di Trento. Avocetta, 25: 113.

Cambi D., 1979 - Contributo allo studio sulla biologia riproduttiva e sulla distribuzione di Sylvia nisoria (Bigia padovana) in Italia. Rivista italiana di Ornitologia, 49: 208-229.

Cambi D., 1982 - Ricerche ornitologiche in Provincia di Foggia (zone "umide" e Gargano) dal 1964 al 1981. Rivista italiana di Ornitologia, 52: 137-153.

Capizzi D., Baccetti N. \& Sposimo P., 2010 - Prioritizing rat eradication on islands by cost and effectiveness to protect nesting seabirds. Biological Conservation, 143 (7): 1716-1727.

Casale F. \& Brambilla M., 2009 - Averla piccola. Ecologia e Conservazione. Fondazione Lombardia per l'Ambiente e Regione Lombardia, Milano.

Casale F. \& Brambilla M., 2010 - L'avifauna nidificante negli ambienti aperti del Parco Nazionale della Val Grande. Ente Parco Nazionale della Val Grande e Fondazione Lombardia per l'Ambiente.

Casale F., Movalli C., Bionda R., Laddaga L., Mosini A. \& Piana M., 2013 - Gli uccelli del Parco Nazionale della Val Grande e delle aree limitrofe (Verbano Cusio Ossola, Piemonte, Italia). Rivista italiana di Ornitologia, 83: 3-52.

Cattadori I.M., Ranci-Ortigosa G., Gatto M. \& Hudson P.J., 2003 - Is the rock partridge Alectoris graeca saxatilis threatened in the Dolomitic Alps? Animal Conservation, 6: 71-81.

Cauli F. \& Aradis A., 2012 - Dati preliminari sullo status dell'Albanella minore, Circus pygargus, nel Lazio. Rivista italiana di Ornitologia, 82: 266-268.

Ceccolini G., Cenerini A. \& Sarà M., 2006 - Il Capovaccaio, specie prossima all'estinzione? In: Salvati dall'Arca. Fraissinet M. \& Petretti F. (eds.). Alberto Perdisa Editore, Bologna: 351-365.

Ceresa F., Bogliani G., Pedrini P. \& Brambilla M., 2012 The importance of key marginal habitat features for birds in farmland: an assessment of habitat preferences of Red-backed Shrikes Lanius collurio in the Italian Alps. Bird Study, 59: 327-334.

Chiatante G.P., Brambilla M. \& Bogliani G., 2014 Spatially explicit conservation issues for threatened bird species in Mediterranean farmland landscapes. Journal for Nature Conservation, 22: 103-112.

Colaone S., Fanchin B., Giusto A., Guenzani W., Saporetti F. \& Zarro T., 2010 - Censimento e playback di una popolazione di Civetta in provincia di Varese. Rivista italiana di Ornitologia, 80: 79-86.

Corso A., 2005 - Avifauna di Sicilia, L'Epos.

Cova C., 1965 - Ornitologia pratica. Edizioni Hoepli, Milano.

Cramp S., 1985 - The Birds of the Western Palearctic. Oxford University Press, Oxford. Volume IV.

Cramp S., 1988 - The Birds of the Western Palearctic. Oxford University Press, Oxford, V.

Cramp S., 1992 - The Birds of the Western Palearctic. Oxford University Press, Oxford, VI.

Cramp S., 1993 - The Birds of the Western Palearctic. Oxford University Press, Oxford, VII.

Cramp S. \& Simmons K.E.L., 1977 - The Birds of the Western Palearctic. Oxford University Press, Oxford, I.

Cramp S. \& Simmons K.E.L., 1980 - The Birds of the Western Palearctic. Oxford University Press, Oxford, II.

Cramp S. \& Simmons K.E.L., 1983 - The Birds of the Western Palearctic. Oxford University Press, Oxford, III.

Cramp S. \& Perrins C.M. (eds.), 1994a - Handbook of the Birds of Europe, the Middle East and North Africa: The Birds of the Western Palearctic. Volume 8 Crows to Finches. Oxford University Press.

Cramp S. \& Perrins C.M., 1994b - The Birds of the Western Palearctic. Oxford University Press, Oxford, IX.

Decandia L., 2015 - Studio della variabilità genetica nel Grifone sardo (Gyps fulvus) attraverso l'analisi del DNA mitocondriale. Tesi di dottorato.

De Franceschi P., 1988 - La situazione attuale dei Galliformi in Italia. Ricerche recenti o ancora in corso. Problemi di gestione e prospettive per il futuro. Supplemento alle Ricerche di Biologia della Selvaggina, XIV: 129-168. 
De Franceschi P., 1994 - Status, geographical distribution and limiting factors of Hazel Grouse in Italy. Gibier faune sauvage, 11 (2): 141-160.

De Franceschi P. \& De Franceschi G., 2006 - Il Gallo cedrone ed altri tetraonidi alpini. In: Salvati dall'Arca. Fraissinet M. \& Petretti F. (a cura di). Alberto Perdisa Editore, Bologna: 489-503.

Dinetti M., 2007 - I Passeri Passer spp. nelle aree urbane e nel territorio in Italia. Distribuzione. Densità e status di conservazione: una review. Ecologia Urbana, 19 (1): 11-42.

Dinetti M. \& Ascani P., 1990 - Atlante degli uccelli nidificanti nel Comune di Firenze. GE 9, Firenze.

Di Vittorio M., 2006 - L'Aquila del Bonelli. In: Salvati dall'Arca. Fraissinet M. \& Petretti F. (a cura di). Alberto Perdisa Editore, Bologna: 407-424.

Di Vittorio M., Seminara S. \& Campobello D., 2000 Aquila di Bonelli, status e biologia riproduttiva in Sicilia. Rivista italiana di Ornitologia, 70: 129-137.

Donald P.F, Sanderson F.J., Burfield I.J., Bieman S.M., Gregory R.D. \& Waliczky Z., 2007 - International Conservation Policy Delivers Benefits for Birds in Europe. Science, 317: 810-813.

Donazar J.A., Palacios C.J., Gangoso L., Ceballos O., Gonzalez M.J. \& Hiraldo F., 2002 - Conservation status and limiting factors in the endangered population of Egyptian vulture (Neophron percnopterus) in the Canary Islands. Biological Conservation, 107: 89-97.

Dougall T.W., Holland P.K., Mee A. \& Yalden D.W., 2005 - Comparative population dynamics of Common Sandpipers Actitis hypoleucos: living at the edge. Bird Study, 52: 80-87.

Evans D. \& Arvela M., 2011 - Assessment and reporting under Article 17 of the Habitats Directive. Explanatory Notes \& Guidelines for the period 2007-2012. Final Draft July 2011. European Topic Centre on Biological Diversity, Paris.

Fasano S. \& Aluigi A., 2012 - Dati preliminari sulla densità riproduttiva di Calandro, Anthus campestris, e Magnanina comune, Sylvia undata, nel Parco del Beigua e nella ZPS "Beigua-Turchino" (GE-SV). Rivista italiana di Ornitologia, 82: 164-166.

Fasce P., 1988 - Censimento dell'Aquila reale nelle alpi occidentali italiane: metodologia e problemi. In: Atti del I Seminario Italiano sui censimenti faunistici. Urbino, 21-22 settembre 1982. Pandolfi M. \& Frugis S. (eds). Istituto di Scienze morfologiche, Università di Urbino: 246-249.

Fasce P. \& Fasce L., 2003 - L'aquila reale Aquila chrysaetos, in Italia: un aggiornamento sullo status della popolazione. Avocetta, 27: 10-13.

Fasce P, Fasce L., 2007 - Stato delle ricerche sull'Aquila reale in Italia. In: Aquila reale, Lanario e Pellegrino nell'Italia peninsulare. Stato delle conoscenze e problemi di conservazione. Magrini M., Perna P. \& Scotti M. (eds.). Atti del Convegno, Serra San Quirico (Ancona), 26-28 marzo 2004. Parco regionale Gola della rossa e di Frasassi: 25-35.

Fasce P., Fasce L., Villers A., Bergese F. \& Bretagnolle V., 2011 - Long-term breeding Demography and density dependance in an increasing population of Golden Eagle Aquila chrysaetos. Ibis, 153 (3): 581591.

Fraissinet M., 2008 - La frequentazione urbana delle specie del Genere Falco in Italia e in Europa. Una monografia. Ecologia urbana, 20 (2): 29-56.

Frederiksen M. \& Bregnballe T., 2000 - Evidence for Density-Dependent Survival in Adult Cormorants from a combined analysis of recoveries and resightings. Journal of Animal Ecology, 69: 737752.

Fulco E., 2014 - Densità riproduttiva del Picchio rosso mezzano Dendrocopos medius in una cerreta della Basilicata e rapporti di abbondanza con le altre specie di Piciformes. In: Atti XVI Convegno Italiano di Ornitologia. Cervia (RA), 22-25 settembre 2011. Tinarelli R., Andreotti A., Baccetti N., Melega L., Roscelli F., Serra L. \& Zenatello M., (eds). Scritti, studi e Ricerche di Storia Naturale della Repubblica di San Marino: 365-366.

Gagliardi A., Carlini E., Chiarenzi B., Cucé L., Macchi S., Martinoli A., Masseroni E., Preatoni D., Spada M., Vigorita V., Wauters L. \& Tosi G., 2014 - I Galliformi alpini in Lombardia: risultati dei primi due anni di monitoraggio su scala regionale. In: Atti XV Convegno Italiano di Ornitologia. Cervia (RA), 22-25 settembre 2011. Tinarelli R., Andreotti A., Baccetti N., Melega L., Roscelli F., Serra L. \& Zenatello M. (eds). Scritti, studi e Ricerche di Storia Naturale della Repubblica di San Marino: 373-376.

Gagliardi A., Guenzani W., Preatoni D.G., Saporetti F. \& Tosi G., 2007 - Atlante Ornitologico Georeferenziato della provincia di Varese. Uccelli nidificanti 20032005. Provicia di Varese, Museo Insubrico di Storia Naturale di Induno Olona e Università dell'Insubria di Varese.

Gaudiano L., Marra M., Talamo V., Sorino R. \& Corriero G., 2014 - Contributo alla conoscenza dello status e distribuzione del Picchio rosso mezzano Dendrocopos medius nel Parco Nazionale del Gargano (Puglia, Italia). In: Atti XVI Convegno Italiano di Ornitologia. Cervia (RA), 22-25 settembre 2011. Tinarelli R., Andreotti A., Baccetti N., Melega L., Roscelli F., Serra L. \& Zenatello M., (eds). Scritti, studi e Ricerche di Storia Naturale della Repubblica di San Marino: 168170.

Giunchi D. \& Pollonora E., 2007 - Monitoraggio della popolazione di Occhione nel sito SIC/ZPS Medio Taro. Consorzio Parco Fluviale del Taro, Relazione inedita.

Grieco F., 1999 - Nest-sites limitation and colony development in tree-nesting great cormorants. Waterbirds, 22: 417-423.

Grussu M., Medda M. \& Asuni V., 2006 - Status del Nibbio reale e del nibbio bruno in Sardegna. In: Status e conservazione del Nibbio reale (Milvus milvus) e Nibbio bruno (Milvus migrans) in Italia e in Europa meridionale. Atti del Convegno, S. Maria del Mercato, Serra San Quirico (Ancona), 11-12 marzo 2006. Parco regionale Gola della rossa e di Frasassi: 3839. 
Guerrieri G., Castaldi A. \& Biondi M., 2001 - Status e selezione di habitat riproduttivo della Cappellaccia, Galerida cristata, nella Riserva Naturale Statale del Litorale Romano. Uccelli d'Italia, Numero Speciale, 26: 109-113.

Guerrieri G., D’Amelia D., De Vita S. Santucci B., 1996 Le comunità ornitiche di steppa nella fascia costiera del Lazio. In: L'avifauna degli ecosistemi di origine antropica. Associazione Studi Ornitologici Italia Meridionale, Napoli: 120-123.

Guerrieri G., Miglio M. \& Santucci B., 2007 - Riproduzione del Porciglione Rallus aquaticus nel Parco Regionale del Sirente-Velino (Appennino centrale). Uccelli d'Italia, 32: 66-70.

Gustin M., Brambilla M. \& Celada C. (eds.), 2009 - Valutazione dello stato di Conservazione dell'avifauna italiana. Non-Passeriformes e Passeriformes dell'Allegato I della Direttiva Uccelli. Ministero dell'Ambiente e della Tutela del Territorio e del Mare, Lega Italiana Protezione Uccelli (LIPU).

Gustin M., Brambilla M. \& Celada C. (eds), 2010a - Valutazione dello Stato di Conservazione dell'avifauna italiana. Volume I. Non-Passeriformes. Ministero dell'Ambiente e della Tutela del Territorio e del Mare, Lega Italiana Protezione Uccelli (LIPU).

Gustin M., Brambilla M. \& Celada C. (eds). 2010b - Valutazione dello stato di conservazione dell'avifauna italiana. Volume II. Passeriformes. Ministero dell'Ambiente e della Tutela del Territorio e del Mare, Lega Italiana Protezione Uccelli (LIPU).

Hagemeijer E.J.M. \& Blair M.J., 1997 - The EBCC Atlas of European Breeding Birds: their distribution and abundance. $T \& A D$ Poyser, London.

Imperio S., Bionda R., Viterbi R. \& Provenzale A., 2013 - Climate change and human disturbance can lead to local extinction of Alpine Ptarmigan: new insight from the western Italian Alps. Plos One, 8 (11): e81598.

Inigo A., Barov B., Orhun C. \& Gallo-Orsi U., 2008 Species action plan for the Egyptian Vulture Neophron percnopterus in the European Union, SEO/BirdLife International.

Jørgensen P.S., Böhning-Gaese K., Thorup K., Tøttrup A.P., Chylarecki P., Jiguet F., Lehikoinen A., Noble DG, Reif J., Schmid H., van Turnhout C., Burfield I.J., Foppen R., Voříšek P., Van Strien A., Gregory R.D. \& Rahbek C., 2015 - Continent-scale global change attribution in European birds - combining annual and decadal time scales. Global Change Biology. doi: 10.1111/gcb.13097, http://onlinelibrary.wiley.com/ doi/10.1111/gcb.13097/full

Katzner T., Milner-Gulland E.J. \& Bragin E., 2006 - Using modelling to improve monitoring of structured populations: are we collecting the right data? Conservation Biology, 21: 241-252.

Kauffman M.J., Winifred F.F. \& Linthicum J., 2003 - Estimation of habitat-specific demography and population growth for Peregrine falcons in California. Ecological Applications, 13: 1802-1816.

Keller V., 2009 - The Goosander Mergus merganser population breeding in the Alps and its connections to the rest of Europe. Wildfowl Special Issue, 2: 60-73.
Lacy R.C., Borbat M. \& Pollak J.P., 2009 - Vortex: a stochastic simulation of the extinction process. Version 9.99. Chicago Zoological Society, Brookfield, IL, USA.

Laiolo P., Rolando A., Bortolin I. \& Valsania V., 1999 Observations on the breeding biology of Choughs Pyrrhocorax pyrrhocorax and Alpine choughs $P$. graculus in the Alps. Avocetta, 23: 106.

La Mantia T., Bonaviri L. \& Massa B., 2014 - Ornithological communities as indicators of recent transformations on a regional scale: Sicily's case. Avocetta, 38: 67-81.

Lo Valvo M., La Mantia T. \& Massa B., 1985 - Bird population of Palermo's urban and suburban areas. Bollettino di Zoologia, 52: 347-354.

Maestri F. \& Voltolini L., 1994 - The population of the Nutcracker Nucifraga caryocatactes in the Stonepine-wood of Val Martello (Rhaetian Alps): census and ecology. Atti del Museo Regio di Scienze naturali, Torino: $335-347$.

Magrini M. \& Perna P., 2007 - Riepilogo ed analisi delle conoscenze sullo status delle popolazioni di Aquila reale, Lanario e Pellegrino nell'Italia peninsulare. In: Aquila reale, Lanario e Pellegrino nell'Italia peninsulare. Stato delle conoscenze e problemi di conservazione. Atti del Convegno, Serra San Quirico (Ancona), 26-28 marzo 2004. Magrini M., Perna P. \& Scotti M., (eds). Parco regionale Gola della rossa e di Frasassi: 133-139.

Magrini M., Perna P., Angelini J., Armentano L. \& Gambaro C., 2013 - Andamento della Popolazione di Aquila reale Aquila chrysaetos in un'area dell'Appennino centrale tra il 1979 e il 2012. In: Atti Secondo Convegno Italiano Rapaci diurni e Notturni, Treviso, 12-13 ottobre 2012. Mezzavilla F. \& Scarton F., (a cura di). Associazione Faunisti Veneti, Quaderni Faunistici, 3: 188-196.

Marchesi L., Pedrini P. \& Sergio F., 2003 - Densità di sei specie di rapaci notturni nel Parco Naturale Adamello-Brenta (Alpi Centrali, TN). Avocetta, 27: 165.

Martelli D., 1997 - Revisione critica delle conoscenze sull'Albanella minore in Italia. Supplemento alle Ricerche di Biologia della Selvaggina, XXVII: 601610.

Martelli D. \& Rigacci L., 2003 - Parametri riproduttivi del Lanario Falco biamicus feldeggii nell'Appennino emiliano. Avocetta, 27: 21.

Mascara R., 2014 - Stato dell'Aquila di Bonelli Hieraaetus fasciatus nella Sicilia centro -meridionale. In: Atti XVI Convegno Italiano di Ornitologia. Cervia (RA), 22-25 settembre 2011. Tinarelli R., Andreotti A., Baccetti N., Melega L., Roscelli F., Serra L., Zenatello M., (eds). Scritti, studi e Ricerche di Storia Naturale della Repubblica di San Marino: 591-592.

Massa B., 1981 - Primi dati sulla nicchia ecologica di cinque Silvidi (Genere Sylvia) in Sicilia. Rivista italiana di Ornitologia, 51: 167-178.

Massa B., 1985 - Atlante degli uccelli nidificanti in Sicilia (1979-1983). Atlas Faunae Siciliae Aves. Naturalista siciliano. Numero speciale, 9: 197-198. 
Massa B. \& La Mantia T., 2010 - The decline of groundnesting birds in the agrarian landscape of Italy. Revue Ecologie (Terre Vie), 65: 73-90.

Massa B., Lo Valvo F., Siracusa M. \& Ciaccio A., 1991 Il Lanario in Italia: status, biologia, tassonomia. Naturalista siciliano, 15: 27-63.

MATTM \& ISPRA., 2012 - Linee guida per le regioni e le province autonome in materia di monitoraggio delle specie e degli habitat di interesse comunitario. Valutazione e rendicontazione ai sensi dell'Art. 17 della Direttiva Habitat Bozza, maggio 2012.

Maurino L., 2012 - Analisi dei censimenti primaverili di Coturnice, Alectoris graeca, nel Parco Naturale Val troncea. Rivista italiana di Ornitologia, 82: 187-189.

Maurino L., Probo M., Gorlier A. \& Lonati M., 2013 Status of rock partridge Alectoris graeca saxatilis in val Troncea Regional Park (Piedmont, north-west Italy). Avocetta, 37:129-132.

Meriggi A., Brangi A., Pella F., Zaccaroni M., Vidus Rosin A., Gandini S., Greco C. \& Dessì Fulgheri F., 2007 - Miglioramenti ambientali e presenza della piccola selvaggina. In: Valorizzazione agro-forestale e faunistica dei territori collinari e montani. Istituto Nazionale Fauna Selvatica, Ministero delle politiche agricole alimentari e forestali \& Sterna: 191-220.

Mezzavilla F. \& Bettiol K., 2007 - Nuovo atlante degli uccelli nidificanti in provincia di Treviso (2003-2006). Associazione Faunisti Veneti.

Minganti A., Carpaneto G. \& Riga F., 2013 - Status passato e attuale del Nibbio reale Milvus milvus e del Nibbio bruno Milvus migrans nidificanti nei monti della Tolfa (Italia centrale). In: Atti Secondo Convegno Italiano Rapaci diurni e Notturni, Treviso, 12-13 ottobre 2012. Mezzavilla F. \& Scarton F., (eds). Associazione Faunisti Veneti, Quaderni Faunistici, 3: 46-51.

Mingozzi T., Boano G. \& Pulcher C., 1988 - Atlante degli uccelli nidificanti in Piemonte e Val d'Aosta 19801984. Monografie del Museo regio di Scienze naturali di Torino VIII.

Mongini E., Marchetti C. \& Baldaccini N.E., 1988 - Inchiesta sulla distribuzione, la consistenza ed i caratteri delle colonie di Topino Riparia riparia. Avocetta, 12: 83-94.

Monti M., 1845 - Ornitologia Comense. Catalogo degli uccelli della Città, Provincia e Diocesi di Como. 1843. Figli di C.A. Ostinelli, Como.

Murgia C., Monni A. \& Marras N., 1988 - Primi dati sulla riproduzione dell'Astore, Accipiter gentilis, in Sardegna con note sulla sua distribuzione e consistenza nella provincia di Cagliari. Rivista italiana di Ornitologia, 58: 142-148.

Nardelli R., Andreotti A., Bianchi E., Brambilla M., Brecciaroli B., Celada C., Dupré E., Gustin M., Longoni V., Pirrello S., Spina F., Volponi S. \& Serra L., 2015 Rapporto sull'applicazione della Direttiva 147/2009/ $\mathrm{CE}$ in Italia: dimensione, distribuzione e trend delle popolazioni di uccelli (20082013). ISPRA, Serie Rapporti, 219/2015.

Newton I., Davis P.E. \& Davis J.E., 1989 -Age of first breeding, dispersal and survival of Red Kites Milvus milvus in Wales. Ibis, 131: 16-21.
Novelletto A. \& Petretti F., 1980 - Ecologia dell'Aquila reale negli Appennini. Rivista italiana di Ornitologia, 50: 127-142.

Nummi P. \& Pöysä H., 1995 - Breeding success of ducks in relation to different habitat factors. Ibis, 137: 145150.

Oro D., Genovart X., Ruiz X., Jimenez J. \& Gans-Garcia J. 1996 - Differences in diet, population size and reproductive performance between two colonies od $\mathrm{Au}-$ douin's Gull Larus audouinii affected by a trawling moratium. Journal of Avian Biology, 27: 245-251.

Palumbo G. \& Gallo-Orsi U., 1999 - Management statement Corso-sardinian Goshawk Accipiter gentilis arrigonii. BirdLife International: 1-6.

Pandolfi M. \& Tanferna A., 2001 - Population dynamics and phylopatry aspects in Montagu's Harrier (Circus pygargus) in central Italy. $4^{\text {th }}$ Eurasian congress on raptors, Seville 25-29 September 2001. Abstracts: 139.

Pandolfi M. \& Tanferna A. 2009 - Long-term change in population size and reproductive Parameters of Montagu's Harrier Circus pygargus in Italy. Journal of Raptor Research, 43: 155-159.

Pedrini P., Caldonazzi M. \& Zanghellini S. (eds.), 2005 Atlante degli uccelli nidificanti e svernanti in provincia di Trento. Museo Tridentino di scienze naturali, Trento. Studi Trentini di Scienze naturali, Acta Biologica 80 , supplemento 2 .

Pellegrini M., De Sanctis A., Altea T., Allegrino C., Civitarese S., Di Francesco N., Di Marzio M., Di Tizio L., Liberatore M., Ponziani V. \& Ricci F., 2014 - Il monitoraggio del Nibbio reale Milvus milvus nella Rete Natura 2000 della regione Abruzzo. In: Atti XVI Convegno Italiano di Ornitologia. Cervia (RA), 22-25 settembre 2011. Tinarelli R., Andreotti A., Baccetti N., Melega L., Roscelli F., Serra L. \& Zenatello M., (eds). Scritti, studi e Ricerche di Storia Naturale della Repubblica di San Marino: 200-202.

Peronace V., Cecere J., Gustin M. \& Rondinini C., 2012 Lista Rossa 2011 degli Uccelli nidificanti in Italia. Avocetta, 36 (1-2): 11-58.

Pirovano A., Sacchi R., Vidus Rosin A., Trespioli G. \& Pinoli G., 2014 - Monitoraggio e selezione dell'habitat del Succiacapre Caprimulgus europaeus nel Parco Pineta di Appiano Gentile-Lombardia. In: Atti XVI Convegno Italiano di Ornitologia. Cervia (RA), 22-25 settembre 2011. Tinarelli R., Andreotti A., Baccetti N., Melega L., Roscelli F., Serra L. \& Zenatello M (a cura di). Scritti, studi e Ricerche di Storia Naturale della Repubblica di San Marino: 205-206.

Quaglierini A., 2006 - Ecologia riproduttiva del cuculo Cuculus canorus in una zona umida dell'Italia Centrale. Avocetta, 30: 33-39.

Rassati G., 2011 - The Hazel grouse, Bonasia bonasia, in the sample areas in the Carnic Alps (FVG, North eastern Italy). Rivista italiana di Ornitologia, 81: 133.143.

Rassati G., 2013 - Distribution and abundance of the Eagle Owl Bubo bubo in Carnia, Canal del Ferro and Valcanale (eastern Alps, Friuli-Venezia Giulia, NE Italy). In: Atti Secondo Convegno Italiano Rapaci diurni e Notturni, Treviso, 12-13 ottobre 2012. Mezzavilla F. \& Scarton F., (eds). Associazione Faunisti Veneti, Quaderni Faunistici, 3: 305-310. 
Ravasini M., 1995 - L'avifauna nidificante nella Provincia di Parma (1980-1995). Editoria Tipolitotecnica. Sala Baganza: 172-174.

Real J. \& Mañosa S., 1997 - Demography and conservation of western European Bonelli's Eagle (Hieraaetus fasciatus) populations. Biological Conservation, 79: 59-66.

Reid J.M., Bignal E.M., Bignal S., McCracken D.I. \& Monaghan P., 2003 - Age-specific reproductive performance in red-billed choughs Pyrrhocorax pyrrhocorax: patterns and processes in a natural population. Journal of Animal Ecology, 72: 765-776.

Rete Rurale Nazionale, Lipu 2015 - Uccelli comuni in Italia. Aggiornamento degli andamenti di popolazione e del Farmland Bird Index per la Rete Rurale Nazionale dal 2000 al 2014.

Ristow D., Scharlau W. \& Wink M., 1989 - Population Structure and Mortality of Eleonora's Falcon Falco eleonorae. In: Raptors in the Modern World. Meyburg B.U. \& Chancellor R.D. (eds.). WWGBP: Berlin, London \& Paris.

Ristow D. \& Wink M., 1985 - Breeding success and conservation management of Eleonora's Falcon. ICBP Technical Publication, 5: 147-152.

Rizzolli F., Sergio F., Marchesi L. \& Pedrini P., 2005 Density, productivity, diet and Population status of the Peregrine Falcon Falco peregrinus in the Italian Alps. Bird Study, 52: 188-192.

Salvati L., Manganaro A. \& Fattorini S., 1999 - Distribuzione, densità, biologia riproduttiva e dieta di una popolazione suburbana di gheppio, Falco tinnunculus, a Roma. Rivista italiana di Ornitologia, 69: 115-122.

Salvo G., 1997 - Movimenti della Calandra Melanocorypha calandra in Sicilia. Avocetta, 21: 69.

Salvo G., 2001 - Andamento riproduttivo e dinamica della popolazione di Lanario Falco biarmicus nella Sicilia centro-meridionale. Avocetta, 25: 66.

Salvo G., 2003 - Densità dell'Assiolo Otus scops in Sicilia meridionale. Avocetta, 27: 122.

Sanderson F.J., Pople R.G., Ieronymidou C., Burfield I.J., Gregory R.D., Willis S.G., Howard C., Stephens P.A., Beresford A.E. \& Donald P.F., 2016 - Assessing the performance of EU nature legislation in protecting target bird species in an era of climate change. Conservation Letters, 9: 172-180.

Sarà M. \& Di Vittorio M., 2003 - Factors influencing the distribution, abundance and nest site selection of an endangered Egyptian Vulture (Neophron percnopterus) population. Animal Conservation, 6 (4): 317-328.

Sarà M. \& Zanca L., 2006 - Status del Nibbio reale e del Nibbio bruno in Sicilia. In: Status e conservazione del Nibbio reale (Milvus milvus) e Nibbio bruno (Milvus migrans) in Italia e in Europa meridionale. Atti del Convegno, S. Maria del Mercato, Serra San Quirico (Ancona), 11-12 marzo 2006. Parco regionale Gola della rossa e di Frasassi: 37.

Schiassi S., Battaglia A., Bonora M., Campora M., Cottalasso R., Del Chiaro L., Mendi M., Pastorino A., Pedrelli M., Ricci U., Sesti L. \& Nardelli R., 2013 - Monitoring of the Golden Eagle Aquila chrysaetos breeding pairs in the northern Appennines (1997-2012).
In: II Convegno Italiano Rapaci diurni e notturni. Mezzavilla F. e Scanton F. (a cura di). Associazione Faunisti Veneti: 179-187.

Schmid H., Luder R., Naef-Daenzer B., Graf R. \& Zbinden N., 1998 - Atlas des Oiseaux nicheurs de Suisse. Station ornithologique Suisse, Sempach.

Sergio F., Pedrini P. \& Marchesi L., 2003 - Reconciling the dichotomy between single species and ecosystem conservation: black kites (Milvus migrans) and eutrophication in pre-Alpine lakes. Biological Conservation, 110: 101-111.

Sorace A., 2011 - Le comunità ornitiche degli ambienti aperti della Sila grande (Calabria) con note sull'apparente contrazione demografica di Saxicola rubetra, Regulus regulus e Carduelis spinus. Avocetta, 35: 5360.

Soutullo A., Lopez-Lopez P. \& Urios V., 2008 - Incorporating spatial structure and stochasticity in endangered Bonelli's eagle's population models: Implications for conservation and management. Biological Conservation, 141: 1013-1020.

Tellini Florenzano G., Arcamone E., Baccetti N., Meschini E. \& Sposimo P. (eds.), 1997 - Atlante degli uccelli nidificanti in e svernanti in Toscana (1982-1992). Quaderni del Museo di Storia Naturale di Livorno. Monografie, 1.

Tellini Florenzano G. \& Lapini L., 1999 - Distribution and habitat of the Dartford Warbler Sylvia undata in the Eastern Tuscany. Avocetta, 23: 32-36.

Tinarelli R., Casini L., Grussu M., Magnani A., Serra L. \& Zenatello M., 2005 - Breeding Avocet in Italy. Wader Study Group, 107: 104-107.

Tye A., Christodoulou-Davies C., Papazoglou C. \& Apostolidou M., 2014 - Setting favourable reference values for annex I bird species at Oroklini marsh as part of the LIFE project: "Restoration and Management of Oroklini Lake SPA in Larnaca, Cyprus". BirdLife Cyprus.

Volponi S., Beraudo P.L., Basso M., Caula B., Cosolo M., Emiliani E., Gagliardi A., Gola L., Panzarin L., Sponza S., Tinarelli R., Albanese G., Bacchi E., Bartolini A., Brunelli M., Calvario E., Carini R., Carotenuto L., Ciaccio A., Fasola M., Gelati A., Grattini N., Ientile R., La Grua G., Mancini V., Pezzo F., Puglisi L., Re A., Sarrocco S., Trainito E. \& Zini C., 2014 - Censimento nazionale delle colonie di Cormorano Phalacrocorax carbo sinesis: anno 2011. In: Atti XVI Convegno Italiano di Ornitologia. Cervia (RA), 22-25 settembre 2011. Tinarelli R., Andreotti A., Baccetti N., Melega L., Roscelli F., Serra L., Zenatello M., (eds). Scritti, studi e Ricerche di Storia Naturale della Repubblica di San Marino: 483-485.

Whitfield D.P., Fielding A.H., McLeod D.R.A. \& Haworth P.F. 2004 - The effects of persecution on age of breeding and territory occupation in golden eagles in Scotland. Biological Conservation, 118, 249-259.

Yeatman Berthelot D., 1995 - Nouvel Atlas des Oiseaux nicheurs de France. Société Ornithologique de France.

Zocchi A., 1992 - Dinamica della popolazione di aquila reale Aquila crhysaetos nell'Appennino centrale nel periodo 1982/1991. Alula, I: 5-10. 